Erschienen in: Engelberg, Stefan/Holler, Anke/Proost, Kristel (Hrsg.):

Sprachliches Wissen zwischen Lexikon und Grammatik. - Berlin, Boston: de

Gruyter, 2011. S. 37-69. (Institut für Deutsche Sprache. Jahrbuch 2011), https://doi.org/10.1515/9783110262339.37

Hans C. Boas

\title{
Zum Abstraktionsgrad von Resultativkonstruktionen
}

\begin{abstract}
Das in diesem Beitrag vorgeschlagene Netzwerk aus Resultativkonstruktionen unterschiedlichen Abstraktionsgrades vereinigt die Vorteile von Goldbergs (1995, 2006) und Boas' (2003a, 2005a) Analysen. So können sowohl die beim Dekodieren relevanten abstrakt-schematischen Konstruktionsinformationen als auch die beim Kodieren wichtigen lexikalisch-spezifizierten Konstruktionsinformationen über semantische, pragmatische, und syntaktische Restriktionen auf der Ebene von Ereignis-Frames in einem einheitlichen Netzwerk erfasst und analysiert werden. Diese einheitliche Analyse bietet zwei entscheidene Vorteile. Erstens vereinfacht die Beschreibung der Distribution von Konstruktionen auf primär semantisch-pragmatischer Ebene die kontrastive Analyse von gleichartigen Konstruktionen in unterschiedlichen Sprachen. Zweitens erfüllt die hier vorgeschlagene Methode auch die in neueren Veröffentlichungen zu Parallelen der Valenzgrammatik und Konstruktionsgrammatik gemachten Vorschläge, unterschiedliche Abstraktionsgrade von Konstruktionen zu berücksichtigen. Ich danke Hans Ulrich Boas, Marc Pierce, Guido Halder, Martin Hilpert, Wolfgang Imo, Klaus Welke, Gert Webelhuth, Alexander Ziem und den Herausgebern für ihre Kommentare und Anregungen.
\end{abstract}

\section{Einleitung}

Spätestens seit Goldbergs (1995) Monographie „Constructions“ sind grammatische Konstruktionen ein (wieder) etabliertes Konzept in der neueren Sprachwissenschaft. Davon zeugen zahlreiche weitere Monographien und Sammelbände, ${ }^{1}$ die Gegenstand empirischer und theoretischer Debatten sind, sowie eine eigens auf Konstruktionen und semantische Frames ausgerichtete Zeitschrift (,Constructions and Frames ${ }^{\circ}$ ) und eine alle zwei Jahre stattfindende internationale Konferenz zur Konstruktionsgrammatik. ${ }^{2}$ Die Ergebnisse konstruktionsgrammatischer Forschung haben nicht nur in die Spracherwerbsforschung (Tomasello 2003; Diessel 2004; Gries/Wulff 2005; Behrens 2009) und die Computerlinguistik (Bergen/Chang 2005; van Trijp 2008; Boas (Hg.) 2009) Eingang gefunden, sondern haben mittlerweile auch andere Theorien beeinflusst. So hat z.B. Ray Jackendoff, einer der prominentesten Vertreter von Chomskys generativer Syntaxtheorie (Chomsky 1981, 1995), das Konzept der Konstruktion als einen wichtigen theoretischen Fortschritt anerkannt, ohne den sich Sprachen nicht vollständig be-

Vgl. Croft (2001), Boas (2003a), Östman/Fried (Hg.) (2005), Fried/Boas (Hg:) (2005), Goldberg (2006), Imo (2007), Barðdal (2008), Bergs/Diewald (Hg) (2008), Hilpert (2008), Iwata (2008), Stefanowitsch/Fischer (Hg.) (2008) u.v.a.

Siehe www.constructiongrammar.org (Stand: 25.10.2010). 
schreiben und analysieren lassen (Jackendoff 1997, 2010). Auch die „HeadDriven Phrase Structure Grammar" (Pollard/Sag 1994) hat das theoretische Konzept der Konstruktion anerkannt und entwickelt sich hin zu einer konstruktionsbasierten Phrasenstrukturgrammatik, der so genannten, ,Signbased Construction Grammar" (siehe Sag 2010; Boas/Sag (Hg.) i.Dr.). ${ }^{3}$

Eine der wichtigsten Thesen konstruktionsgrammatischer Forschung ist, dass es keine klare Trennung zwischen universalgrammatischer Kerngrammatik und Peripherie gibt. So umfasst der relativ flexible Konstruktionsbegriff alle Arten linguistischer Zeichen (d.h. konventionalisierte Paare aus Form- und Bedeutungskomponenten), unabhängig davon wie abstraktschematisch oder konkret diese sind. Dies bedeutet auch, dass es im Prinzip - anders als in anderen gängigen Grammatikmodellen - keine klare Trennung von Grammatik und Lexikon gibt, da derselbe Formalismus zur Beschreibung von Konstruktionen unterschiedlicher Abstraktionsgrade bzw. Komplexitätsstufen benutzt werden kann (vgl. Kay/Fillmore 1999; Boas 2008a, 2008b; Iwata 2008; Sag 2010; Fillmore/Lee-Goldman/Rhodes i.Dr.). Diese Paarung von Form und Bedeutung/Funktion ist in Abbildung 1 dargestellt.

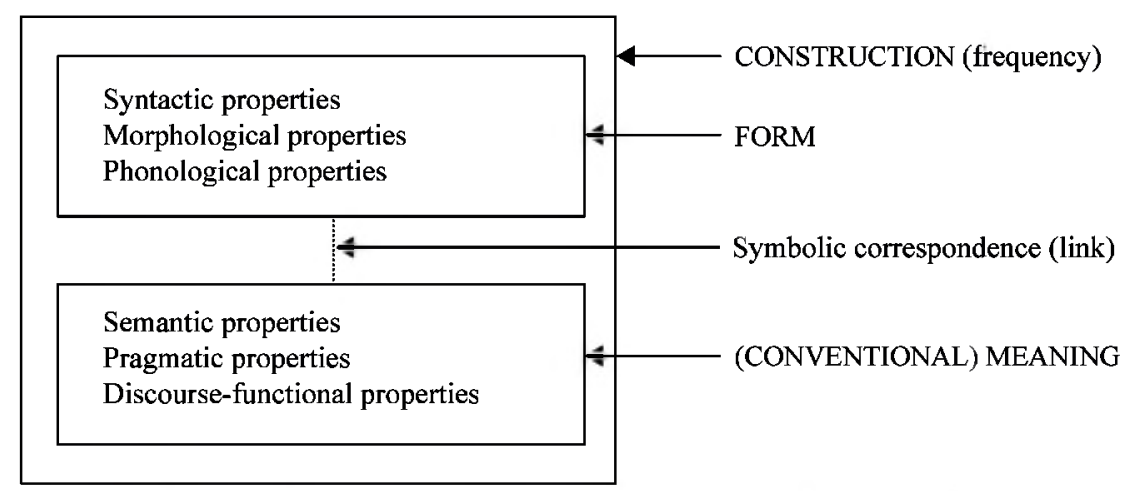

Abb. 1: Beziehung zwischen Form und Bedeutung in der Konstruktionsgrammatik (Croft 2001, S. 18)

Die Konstruktionsgrammatik wurde in den 1980er Jahren ursprünglich nur für die Beschreibung und Analyse des Englischen entwickelt. Ziel war zunächst eine beschreibungsadäquate Theorie einer einzelnen Sprache zu entwickeln, wie Kay/Fillnore (1999, S. 1) klarstellen: „To adopt a constructional approach is to undertake a commitment in principle to account for the entirety of each language. This means that the relatively general patterns of the language, such as the one licensing the ordering of a finite ausiliary verb before its subject in English, often known as SAI, and the highly idiomatic patterns, like kick the bucket, stand on an equal footing as data for which the grammar must account. An explicit grammar that covers the full range of constructions must represent all constructions, of whatever degree of generality or idiomaticity, in a common notation and must provide an explicit account of how each sentence of a language is licensed by a subset of the leaves of the inheritance hierarchy of constructions which constitutes the grammar of that language. "Seit den 1990er Jahren sind die Ideen der Konstruktionsgrammatik auch auf andere Sprachen angewandt worden. Dabei lassen sich einzelsprachliche Studien wie z.B. Fillmore/Kay/O'Connor (1988), Lam- 
Aus dieser einheitlichen Sicht von Sprache ergibt sich, dass es Konstruktionen mit unterschiedlichem Abstraktionsgrad gibt. Der Status von abstrakten Konstruktionen in der Konstruktionsgrammatik ist aber umstritten. Goldberg $(1995,2006)$ postuliert abstrakt-schematische Konstruktionen wie die Resultativkonstruktion in Verbindung mit Lexikoneinträgen von Verben, die Resultativsätze wie z.B. Fritr. bustet den Schaum vom Bier lizenzieren sollen. ${ }^{4}$ Im Gegensatz dazu wird in Boas (2003a, 2005a) die Ansicht vertreten, dass Resultativkonstruktionen nicht immer ausreichend restringiert werden können, was zur Übergenerierung führen kann. So können von der Konstruktion Sätze lizenziert werden, die im tatsächlichen Sprachgebrauch nicht vorkommen oder nicht zulässig sind, wie z.B. Frito inhaliert das Buch vom Regal. Nach der in Boas (2003a, 2005a) vertretenen Auffassung können die so entstehenden Probleme nur dadurch gelöst werden, dass lexikalisch spezifierte Resultativkonstruktionen auf konkret-komplexeren Ebenen postuliert werden, um so Übergenerierungen zu verhindern.

Ziel dieses Beitrags ist es zu zeigen, dass sich die beiden Analysen nicht grundsätzlich gegenseitig ausschließen, sondern dass sie sich innerhalb eines Konstruktionsnetzwerks wie bei Langacker (2000) ergänzen. Die Kombination der beiden Analysen hat zur Folge, dass je nach Konstruktion unterschiedliche Arten von konkret-spezifizierten Informationen gelistet und in einem Konstruktionsnetzwerk bzw. einer Konstruktionshierarchie festgehalten werden müssen, die auch z.T. redundante Informationen enthalten. Die so vorgeschlagene Arbeitsteilung zwischen abstrakt-schematischer Konstruktion und lexikalisch-spezifizierten Konstruktionen verbindet die Vorteile von Goldbergs und Boas' Vorschlägen.

Der Beitrag ist wie folgt gegliedert: Zunächst erläutere ich Goldbergs $(1995,2006)$ Analyse der (englischen) Resultativkonstruktion. Dann zeige ich, dass Goldbergs Analyse eine implizite Trennung zwischen Lexikon und Grammatik annimmt, die zur Folge hat, dass die Beschränkungen, welche die Fusionierung von abstrakten Konstruktionen mit konkreten Lexikoneinträgen von Verben regulieren sollen, nicht immer funktionieren (vgl. Boas 2003b, 2005a). Auf der Basis von Kollokationsanalysen und Frequenzdaten argumentiere ich, dass Konstruktionen auf unterschiedlichen Abstraktionsebenen existieren, die in einer Konstruktionshierarchie miteinander verbunden sind (vgl. Boas 2003a). Außerdem zeige ich, inwieweit

brecht/Lemoine (2005) und Barðdal (2008) von konstrastiven Analysen wie etwa Boas (2003a, 2010b), Hilpert (2008), Iwata (2008) unterscheiden. Zur Kritik einer sprachübergreifenden bzw. universellen Konstruktionsgrammatik siehe Croft (2001).

4 Die Lizenzierung erfolgt natürlich auch durch „Coercion", d.h. durch einen entsprechenden Interpretationsprozess. Goldberg (1995) unterscheidet zwischen so genannten Caused-Motion-Konstruktionen (z.B. Frits, bustet den Schaum vom Bier) und so genannten Resultativkonstruktionen (z.B. Ruth läuft sich die Füße wund). Im Gegensatz dazu folge ich Boas (2003a), der beide Sorten von Konstruktionen als Resultativkonstruktion analysiert, da sie eine parallelinterpretierbare Semantik haben, d.h., sie bezeichnen beide einen Endzustand des postverbalen Objekts. 
lexikalische Einträge von Verben valenz-relevante Informationen enthalten, die über die Lizenzierung von postverbalen Nominal- und Resultativphrasen Auskunft geben können. Ich schließe mit Überlegungen, die zeigen sollen, wie Konstruktionen von unterschiedlichem Abstraktionsgrad mit einer einheitlichen Notation erfasst werden können. Der Großteil der in diesem Beitrag diskutierten Daten stammt aus dem Englischen, weil Goldberg (1995) auf englischen Daten beruht. Um eine erweiterte konstrastive Perspektive zu bieten, bespreche ich aber auch deutsche Resultativkonstruktionen, die zeigen, inwieweit sich die auf dem Englischen basierenden Methoden auf das Deutsche übertragen lassen.

\section{Der Status abstrakter Konstruktionen}

\subsection{Der Konstruktionsbegriff in Goldbergs Theorie}

Anders als der ursprüngliche konstruktionsgrammatische Ansatz von Fillmore und Kay (Fillmore 1988; Fillmore/Kay/O'Connor 1988; Fillmore/Kay 1995; Kay/Fillmore 1999), der Konstruktionen eigentlich nur dann postuliert, wenn diese zumindest partiell unanalysiert sind, schlägt Goldberg $(1995,2006)$ auch solche Einheiten als Konstruktionen vor, die sowohl völlig transparent als auch partiell oder vollständig unanalysierte, nicht-kompositionelle Einheiten sind: „It's constructions all the way down" (Goldberg 2006, S. 18). Nach dieser Auffassung sind alle Form-Bedeutungs-Paarungen als unterschiedliche Konstruktionen anzusehen, wenn sich deren Existenz nicht aus schon vorher existierenden Konstruktionen erklären lässt:

According to Construction Grammar, a distinct construction is defined to exist if one or more of its properties are not strictly predictable from knowledge of other constructions existing in the grammar: $C$ is a construction iff $_{\text {def }} C$ is a form-meaning pair $\left\langle F_{i}, S_{i}\right\rangle$ such that some aspect of $F_{i}$ or some aspect of $S_{i}$ is not strictly predictable from C's component parts or from other previously established constructions. (Goldberg 1995, S. 4)

Any linguistic pattern is recognized as a construction as long as some aspect of its form or function is not strictly predictable from its component parts or from other constructions recognized to exist. In addition, patterns are stored as constructions even if they are fully predictable as long as they occur with sufficient frequency. (Goldberg 2006, S. 5) ${ }^{5}$

Diese Ansicht hat zur Folge, dass Konstruktionen im Prinzip das gesamte Spektrum des Syntax-Lexikon-Kontinuums abdecken, von formal vollständig kompositionellen und semantisch transparenten Strukturen bis hin zu vollständig lexikalisierten und formal fixierten Redewendungen, wie in Tabelle 1 dargestellt.

$\mathrm{Zu}$ Alternativdefinitionen von Konstruktionen siehe z.B. Croft (2001, S. 17-21) und Fried/ Östman (Hg.) (2004, S. 18-23). 


\begin{tabular}{|l|l|}
\hline Morpheme & e.g. pre-, -ing \\
\hline Word & e.g. avocado, anaconda, and \\
\hline Complex word & e.g. daredevil, shoo-in \\
\hline Complex word (partially filled) & e.g. [N-s] (for regular plurals) \\
\hline Idiom (filled) & e.g. going great guns, give the Devil his due \\
\hline Idiom (partially filled) & $\begin{array}{l}\text { e.g. jog < }<\text { someone's }>\text { memory, send } \\
<\text { someone }>\text { to the cleaners }\end{array}$ \\
\hline Covariational Conditional & $\begin{array}{l}\text { The Xer the Yer (e.g. the more you think } \\
\text { about it, the less you understand) }\end{array}$ \\
\hline Ditransitive (double object) & $\begin{array}{l}\text { Subj } \mathrm{V} \mathrm{Obj}, \mathrm{Obj}_{2}(\mathrm{e} . \mathrm{g} . \text { he gave her a fish } \\
\text { taco; he baked her a muffin) }\end{array}$ \\
\hline Passive & $\begin{array}{l}\text { Subj aux } \mathrm{VP}_{\mathrm{PP}}\left(\mathrm{PP}_{\mathrm{by}}\right) \text { (e.g. the armadillo } \\
\text { was hit by a car) }\end{array}$ \\
\hline
\end{tabular}

Tab. 1: Beispiele von Konstruktionen, die sich in Unfang und Komplexität unterscheiden (nach Goldberg 2006, S. 5$)^{6}$

Außer solchen Konstruktionen wie in Tabelle 1 gibt es laut Goldberg auch noch so genannte abstrakt-schematische Konstruktionen, die unabhängig existieren und eine eigene Bedeutung haben. Solche Konstruktionen verbinden ebenfalls eine bestimmte Form mit einer bestimmten Bedeutung/ Funktion, und sie können unter gegebenen Umständen Verben semantische Argumente beifügen, die sie sonst alleine nicht hätten, wie in Beispiel (1).

a. He talked himself blue in the face. (Goldberg 1995, S. 189)

b. Er redete sich dumm und dusselig.

Goldberg behauptet, dass das Verb talk alleine nicht die ganze Bedeutung mitbringt, die es in (1a) zum Ausdruck bringt. Intransitive Verben wie talk haben laut Goldberg keine eigenständige Bedeutung, die den Endzustand von Personen beschreiben, die viel reden. Um dieses Problem zu lösen, postuliert sie Lexikoneinträge, welche die Grundbedeutung von Verben repräsentieren, und zwar im Format der Frame-Semantik von Fillmore (1985): „Lexical entries should make reference to world and cultural knowledge " (Goldberg 1995, S. 28). Der Lexikoneintrag des Verbs talk in (2) verfügt in Goldbergs Ansatz über eine so genannte Partizipantenrolle (den TALKER), die stellvertretend für die Beschreibung der Situation eines Sprechgeschehens steht.

(2) talk $<$ talker $>$

(Goldberg 1995, S. 189)

Die von Goldberg (2006, S. 5) gelisteten Konstruktionen sind entweder Morpheme, einzelne Wörter oder aber abstraktere morpho-syntaktische Konstruktionen, die partiell mit lexikalischen Einheiten gefïllt sind. Darüber hinaus gibt es aber auch konstruktionsgrammatische Analysen, die sich mit anderen Aspekten der Sprache beschäftigen, vgl. z.B. Boas (2004) zur Interaktion von Syntax, Morphologie und Phonologie und Östman (2005), Deppermann (2007) und Günthner (2008) zur Diskurs- bzw. Konversationsanalyse. 


\subsection{Fusionierung abstrakter Konstruktionen mit Verben}

Um die Lizenzierung von Sätzen wie in (1a) zu erklären, behauptet Goldberg (1995, S. 180-198), dass Verben wie talk in (2) mit der unabhängig existierenden abstrakt-schematischen „Resultative Construction“ fusionieren können, die eine bestimmte Bedeutung (X CAUSES Y TO BECOME Z) mit einer bestimmten Form (SUBJ, OBJ, OBL) paart. Die Variablen X, Y und Z in der Bedeutungsbeschreibung der Konstruktion sind als semantische Argumentrollen zu verstehen, d.h. X als AGENT (derjenige, der etwas tut), $\mathrm{Y}$ als PATIENT (derjenige, mit dem etwas gemacht wird) und $Z$ als das RESULT-GOAL (der Endzustand, den der PATIENT nach der vom Verb bezeichneten Aktivität erreicht). Diese Bedeutungen sind, wie in Abbildung 2 dargestellt, mit bestimmten syntaktischen Relationen, nämlich Subjekt, Objekt und Oblique gepaart.

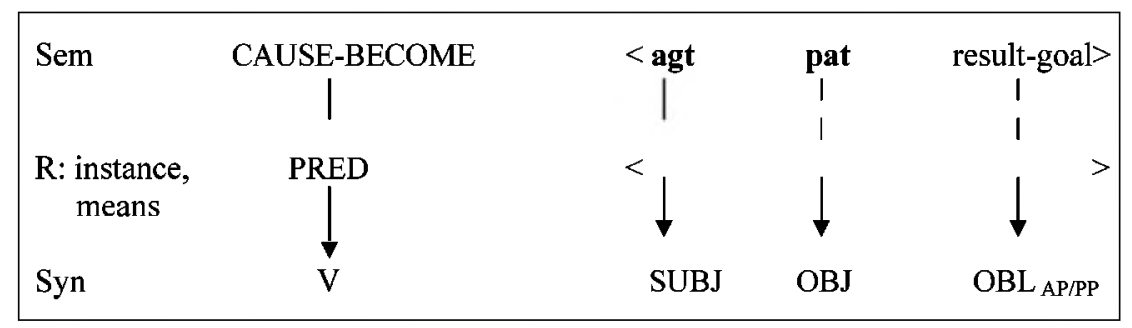

Abb. 2: Resultativkonstruktion (vgl. Goldberg 1995, S. 189)

Die oberste Zeile in Abbildung 2 repräsentiert die Semantik (,Sem') der abstrakt-schematischen Resultativkonstruktion, die eine Verursachungsbeziehung (CAUSE-BECOME) zwischen den Argumentrollen AGENT und PATIENT der Konstruktion beschreibt. Der daraus hervorgehende Resultatszustand wird durch die Argumentrolle RESULT-GOAL dargestellt. Die von oben nach unten führenden Pfeile deuten an, dass die Semantik der Konstruktion mit einer bestimmten syntaktischen Form gepaart ist, die in der untersten Zeile unter „Syn" als Subjekt, Objekt und Oblique aufgeführt ist. Der vielleicht wichtigste Aspekt der Konstruktion ist die Zeile in der Mitte (R: instance, means), in die das Verb (PRED) unter bestimmten Umständen eingefügt werden kann. Wenn ein Verb mit einer Konstruktion fusioniert, dann geschieht das laut Goldberg dadurch, dass die Partizipantenrollen des Lexikoneintrags des Verbs in die mittlere Zeile (PRED) der Konstruktion eingefügt werden. Dieser Vorgang ist jedoch nur möglich, wenn die Partizipantenrolle(n) des Verbs mit der/den Argumentrolle(n) der Konstruktion semantisch kompatibel sind.

Wenn die Rollen des Verbs und der Konstruktion auf diese Weise abgeglichen werden können, dann findet die Fusionierung des Verbs mit der Konstruktion statt. So kann z.B. die Partizipantenrolle TALKER des Verbs 
talk in (2) in die Resultativkonstruktion in Abbildung 2 eingefügt werden, weil diese als eine spezifischere Realisierung der verallgemeinerten Argumentrolle AGENT der Konstruktion interpretiert werden kann und als solche kompatibel mit ihr ist. Somit fusionieren laut Goldberg auch gleichzeitig die Bedeutungen des Verbs und der Konstruktion. ${ }^{7}$ Immer wenn solch eine Fusionierung von Verb und Konstruktion stattfindet, hat die Konstruktion gleichzeitig die Möglichkeit, zusätzliche Argumentrollen beizusteuern. Im Falle von talk kann die Resultativkonstruktion zwei weitere semantische Argumentrollen beisteuern, den PATIENT und das RESULTGOAL. Diese werden dann, genauso wie die AGENT-Rolle, durch die Konstruktion syntaktisch realisiert. Auf diese Art und Weise erklärt Goldberg nicht nur die Lizenzierung der vom Verb nicht-subkategorisierten Konstituenten in Resultativkonstruktionen, sondern auch analoge Phänomene in einer Reihe von anderen Konstruktionen wie der „Ditransitive Construction" und der „,Way Construction". Analoge Vorschläge lassen sich auch zur Lizenzierung von Resultativkonstruktionen im Deutschen wie in (1b) machen (siehe Boas 2002a).

Einer der Vorteile von Goldbergs Analyse liegt darin, dass Verben nur eine prototypische Grundbedeutung haben, weil abstrakte Konstruktionen den Verben durch Fusionierung zusätzliche Argumentstellen beisteuern können. Dies ermöglicht eine systematische Generalisierung auf Konstruktionsebene, wodurch gleichzeitig verhindert werden kann, dass alle syntaktischen Kontexte, in denen ein Verb vorkommen kann, in dessen Lexikoneintrag spezifiziert werden müssen (vgl. Goldberg 1995, S. 224). Ein weiterer Vorteil ist laut Goldberg, dass Konstruktionen unabhängig von der Bedeutung einzelner Verben als Form-Bedeutungs-Paare abstrahierbar sind. Dies wiederum erlaubt eine Reduzierung der Länge und Detailgenauigkeit von Lexikoneinträgen, was wiederum zu minimaleren Lexikoneinträgen führt (vgl. Goldberg 1995, S. 28-30).

\subsection{Probleme mit Goldbergs abstrakt-schematischen Konstruktionen}

Goldbergs abstrakt-schematische Konstruktionen sind zwar in der Lage, mit Verben zu fusionieren und ihnen semantische Argumentrollen beizusteuern, aber dieser Prozess kann nicht immer korrekt restringiert werden, da es nicht immer möglich ist, genaue Kriterien festzulegen, die helfen können zu entscheiden, warum ein bestimmtes Verb mit einer Konstruktion fu-

\footnotetext{
Die Fusionierung von Lexikoneinträgen mit der Resultativkonstruktion unterliegt einer Reihe von semantischen Restriktionen, vgl. Goldberg (1995, S. 193-197), sowie generellen Restriktionen wie dem „Semantic Coherence Principle“ und dem "Correspondence Principle“ (Goldberg 1995, S. 50). Diese Restriktionen sollen die Übergenerierung von nichtattestierten Sätzen verhindern, ähnlich wie so genannte Filter in der generativen Grammatik Chomskys (siehe Boas 2008a).
} 
sioniert, aber ein anderes bedeutungsverwandtes Verb nicht (siehe u.a. Kay 1996, 2005; Nemoto 1998, 2005; Boas 2002b, 2003a, 2007, 2008b). Dies hat u.a. mit der Struktur der Lexikoneinträge bei Goldberg zu tun, welche nicht immer detailgenau die vielen unterschiedlichen Aspekte von Wortbedeutungen mit frame-semantischen Beschreibungen darstellen. So lässt sich z.B. anhand der Lexikoneinträge einiger bedeutungsverwandter Kommunikationsverben wie in (3a)-(3f) nicht genau feststellen, welche Bedeutungsaspekte eines Verbs für die Fusionierung mit einer Konstruktion relevant sind, oder welche einer Fusionierung gar im Wege stehen könnten. Parallele Beobachtungen lassen sich für das Deutsche machen.
a. talk $<$ talker $>$
b. speak $<$ speaker $>$
c. whisper $<$ whisperer $>$
d. grumble $<$ grumbler $>$
e. murmur $<$ murmurer $>$
f. $\operatorname{sigh}<$ sigher $>$

(vgl. Boas 2003a, S. 106)

Obwohl die Lexikoneinträge in (3a)-(3f) in der Lage sind mit einigen Konstruktionen wie der Hauptsatzkonstruktion zu fusionieren (vgl. Miriam \{talked/spoke/whispered/grumbled/murmered/sighed\} (to Joe)), können nicht alle mit anderen Konstruktionen fusionieren. So zeigt z.B. Boas (2003a), dass das Verb talk in (4a) mit der Resultativkonstruktion fusionieren kann, aber nicht andere bedeutungsverwandte Kommunikationsverben wie speak, whisper, grumble, murmur und sigh in (4b)-(4f). Mit den von Goldberg postulierten allgemeinen semantischen Restriktionen, welche die Fusionierung restringieren sollen (siehe Fußnote 7 ), lassen sich unakzeptable Beispiele wie in (4b)-(4f) nicht systematisch ausschließen. Im Deutschen verhält es sich ähnlich (vgl. Boas 2003a, S. 287-289). ${ }^{8}$

(4) a. Miriam talked herself blue in the face.

b. *Miriam spoke herself blue in the face.

c. ?Miriam whispered herself blue in the face.

d. ${ }^{*}$ Miriam grumbled herself blue in the face.

e. *Miriam murmured herself blue in the face.

f. ?Miriam sighed herself blue in the face. (vgl. Boas 2003a, S. 105)

Weitere Schwierigkeiten ergeben sich, wenn es darum geht, die semantische Bandbreite der von der Konstruktion beigesteuerten Argumentrollen zu bestimmen. So zeigt Boas (2003a, 2008a), dass bedeutungsverwandte

Boas (2008b) untersucht auch die Distribution der Verben in (4a)-(4f) in der „Ditransitive Construction" und der so genannten "Way Construction“. Es wird gezeigt, dass die Distribution dieser sechs bedeutungsverwandten Verben sich auch bzgl. dieser beiden Konstruktionen stark unterscheidet, d.h., einige Verben können mit den Konstruktionen fusionieren und andere nicht. 
Verben wie eat (,essen), chew (kauen), devour (,verschlingen) und swallow (,(ver)schlucken') nicht alle dieselben Kategorien von postverbalen Konstituenten zulassen. Wenn man z.B. Goldberg folgend für eat einen Lexikoneintrag wie in (5) annimmt und dieser Eintrag mit einer Reihe von unterschiedlichen Konstruktionen fusionieren kann, um so unterschiedliche Sätze wie in (6a)-(6d) zu lizenzieren, dann könnte man auch erwarten, dass die bedeutungsverwandten Verben in (7)-(9) mit denselben Konstruktionen fusionieren können, was jedoch nicht der Fall ist.

eat: < eater eaten $>$

(6) a. Pat ate.

b. Pat ate his food.

c. Pat ate his food up.

d. Pat ate his plate clean.

(7) a. Pat chewed.

b. Pat chewed his food.

c. Pat chewed his food up.

d. *Pat chewed his plate clean.
a. *Pat devoured.
b. Pat devoured his food.
c. *Pat devoured his food up.
d. *Pat devoured his plate clean.

(9) a. Pat swallowed.

b. Pat swallowed his food.

c. ?Pat swallowed his food up.

d. ${ }^{*}$ Pat swallowed his plate clean. essen: $<$ Esser Gegessenes >

a'. Pat aß.

b'. Pat aß sein Essen.

c'. Pat aß sein Essen auf.

d'. Pat aß seinen Teller leer.

a'. Pat kaute.

b'. Pat kaute sein Essen.

c'. ?Pat kaute sein Essen auf.

d'. *Pat kaute seinen Teller leer.

$a^{\prime}$. *Pat verschlang.

b'. Pat verschlang sein Essen.

c'. *Pat verschlang sein Essen auf.

d'. *Pat verschlang seinen Teller leer.

a'. Pat schluckte.

b'. Pat schluckte sein Essen.

c'. Pat verschluckte sein Essen.

d'. *Pat schluckte/verschluckte seinen Teller leer.

Parallele Beobachtungen lassen sich für das Deutsche machen. Die deutschen Parallelbeispiele belegen einen weiteren wichtigen Punkt, nämlich dass die Resultativphrasen zu einem gewissen Grad konventionalisiert sind und nicht anhand von generellen Prinzipien erfasst werden können. So ist es z.B. im Deutschen normalerweise nicht möglich, die Resultativphrase sauber zusammen mit essen zu verwenden, vgl. (6d'). Goldbergs Fusionierung von Verben und abstrakten Konstruktionen ist auch problematisch, wenn es um die semantisch-pragmatische Restringierung von postverbalen Konstituenten geht. So zeigt z.B. Boas (2003a), dass Verben sehr spezielle Anforderungen bzgl. der Resultativphrasen stellen, wie in den Tabellen 2 und 3 dargestellt. 


\begin{tabular}{|c|c|}
\hline Verb & No. of occur. \\
\hline shoot & 408 \\
\hline kill & 9 \\
\hline strike & 8 \\
\hline make, knock & 3 \\
\hline flatten, kick, smite & 1 \\
\hline
\end{tabular}

Tab. 2: Distribution von Verben mit dead in Resultativkonstruktionen im British National Corpus (BNC) (Boas 2003a, S. 130)

\begin{tabular}{|c|c|}
\hline Verb & No. of occur. \\
\hline stab & 114 \\
\hline beat & 74 \\
\hline batter & 39 \\
\hline frighten & 34 \\
\hline crush & 25 \\
\hline scare & 24 \\
\hline burn & 18 \\
\hline torture & 16 \\
\hline drink, starve & 15 \\
\hline bludgeon, hack & 12 \\
\hline shoot, kick & 11 \\
\hline club & 9 \\
\hline bore, knife, choke & 8 \\
\hline blast, trample, work, worry & 7 \\
\hline love & 6 \\
\hline strangle & 4 \\
\hline dash, poison, kiss & 3 \\
\hline $\begin{array}{l}\text { axe, bayonet, boil, bring, clap, suffocate, kick, } \\
\text { freeze, spear, spray, stone, suck, gun, hammer, } \\
\text { hug, knock, nag, peck, play, rape, shag, sting }\end{array}$ & 2 \\
\hline $\begin{array}{l}\text { annoy, eat, bleed, blend, bug, bully, flog, frit, } \\
\text { cudgel, curse, dance, feed, gas, flog, jog, } \\
\text { laugh, pitchfork, pound, run, schmaltz, scorch, } \\
\text { scratch, seduce, shock, sing, smother, squash, } \\
\text { squeeze, stamp, strike, suffocate, sweat, whip }\end{array}$ & 1 \\
\hline
\end{tabular}

Tab. 3: Distribution von Verben mit to death in Resultativkonstruktionen im BNC (Boas 2003a, S. 131) 
Die Tabellen 2 und 3 sind Teilergebnisse aus der umfangreichen korpusbasierten Studie von Resultativkonstruktionen in Boas (2003a). Um festzustellen, welche Arten von Resultativphrasen mit bestimmten Verben vorkommen können, habe ich die Kollokationen von fünfzig Resultativphrasen (insbesondere Adjektiv- und Präpositionalphrasen) und der damit erscheinenden Verben im British National Corpus untersucht (vgl. Boas 2003a, S. 11-18). Daten wie in Tabelle 2 und 3 belegen, dass Goldbergs abstraktschematische Resultativkonstruktion nie in der Lage ist, systematisch zu bestimmen, welche Arten von Resultativphrasen mit welchen Verben vorkommen können. Dies ist besonders offensichtlich bei Resultativphrasen, die bedeutungsverwandt sind, wie z.B. die Adjektivphrase dead (,tot') und die Präpositionalphrase to death (,zu Tode). Dies lässt sich aber auch für den Rest der von mir untersuchten Resultativphrasen feststellen. Die Datenlage im Deutschen ist sehr ähnlich (siehe Boas 2003a, S. 285-312). Aus diesen Beobachtungen schließe ich, dass abstrakte Resultativkonstruktionen, wenn sie mit Lexikoneinträgen von Verben fusionieren, nicht in der Lage sind, die relativ idiosynkratische Distribution von Resultativphrasen adäquat zu restringieren.

Zusammenfassend lässt sich bzgl. der Interaktion von abstrakt-schematischen Konstruktionen und Lexikoneinträgen Folgendes feststellen: (1) Goldbergs Ansatz ermöglicht es nicht, systematisch zu erklären, warum Resultativkonstruktionen mit einem bestimmten Verb fusionieren können, aber nicht mit einem bedeutungsverwandten Verb. Der Prozess der Fusionierung kann so zur Übergenerierung führen. (2) Der Prozess der Fusionierung von Verben und Konstruktionen ist nicht in der Lage, die semantische, pragmatische und syntaktische Bandbreite von Resultativphrasen zu restringieren, da die dazu nötigen Informationen und Mechanismen fehlen. Diese Schwierigkeiten treffen nicht nur auf Resultativkonstruktionen zu, sondern lassen sich auch bei anderen abstrakten Konstruktionen nachweisen, wie z.B. der "Caused-motion Construction" (vgl. Boas 2002b, 2003b, 2006; Iwata 2005; Kay 2005), der „Ditransitive Construction“ (Nemoto 1998; Kay 2005; Boas 2010a), und der der Lokativalternation unterliegenden Konstruktionen (Michaelis/Ruppenhofer 2001; Boas 2006, 2009, i.Dr.; Nemoto 2005; Iwata 2008).

\section{Lexikalisch-spezifizierte Minikonstruktionen}

Um die oben dargestellten Probleme zu lösen, habe ich in einer Reihe von Publikationen vorgeschlagen, die Lexikoneinträge von Verben genauer zu spezifizieren, um so die Lizenzierung von Resultativkonstruktionen (und anderen nur partiell produktiven Konstruktionen) adäquat zu erfassen (vgl. Boas 2003a, 2005a, 2007, 2008a, 2009). Dieser Alternativansatz sieht eine 
viel bedeutendere Rolle der Frame-Semantik (Fillmore 1985) vor, in dem größerer Wert auf eine genauere Analyse einzelner Verbbedeutungen gelegt wird.

Die Frame-Semantik beruht auf der Idee, dass Wortbedeutungen (fast) immer Bezug auf strukturierte semantische Konzepte, Erfahrungen, Erinnerungen und Weltwissen nehmen - so genannte semantische Frames -, die für die korrekte Benutzung von Wörtern unabdingbar sind (vgl. Petruck 1996; Fillmore 2007; Ziem 2008). Fillmore/Atkins (1992, S. 76-77) beschreiben die zentrale Rolle von Frames wie folgt.

A word's meaning can be understood only with reference to a structured background of experience, beliefs, or practices, constituting a kind of conceptual prerequisite for understanding the meaning. Speakers can be said to know the meaning of the word only by first understanding the background frames that motivate the concept that the word encodes. Within such an approach, words or word senses are not related to each other directly, word to word, but only by way of their links to common background frames and indications of the manner in which their meanings highlight particular elements of such frames.

Als Beispiel sei hier das englische Verb talk (,reden', sprechen') genannt, welches in einer seiner Bedeutungen den so genannten Statement Frame evoziert. ' In FrameNet, einer lexikografischen Onlinedatenbank des Englischen, welche die theoretischen Prinzipien der Frame-Semantik in die Praxis umsetzt (vgl. Fillmore/Johnson/Petruck 2003; Boas 2005b; Ruppenhofer et al. 2006; Fillmore/Baker 2010) ${ }_{,}^{10}$ beschreibt der Statement Frame Szenarien mit unterschiedlichen voneinander abhängigen so genannten Frame Elements, die spezifischere Realisierungen abstrakterer semantischer Rollen wie AGENT, UNDERGOER, INSTRUMENT etc. darstellen. Die Definition des Statement Frames besteht aus den Definitionen der einzelnen Frame Elements sowie ihrer Beziehungen zueinander. Dieser Frame beschreibt Situationen, in denen ein SPEAKER (der Sprecher) einen ADDRESSEE (den Adressaten) mit einer MESSAGE (die Nachricht) anspricht, die auch indirekt durch ein TOPIC (das Thema) ausgedrückt weden kann. Der ADDRESSEE ist diejenige Person, die die Nachricht vom Sprecher erhält. Die MESSAGE ist das vom Sprecher Gesagte. Der SPEAKER ist die wahrnehmende Person, welche die Nachricht produziert, und das Frame Element TOPIC beschreibt generell den Inhalt der Nachricht (vgl. Ruppenhofer et al. 2006).

Die Frame-Semantik vertritt ein feinkörniges Konzept der Polysemie, d.h., Wörter werden entsprechend ihrer Unterbedeutungen beschrieben und analysiert. So aktiviert das Verb talk nicht nur den statement Frame, sondern auch den Chatting und Talking_into Frame. Obwohl alle drei Frames miteinander in Beziehung stehen (alle beinhalten z.B. einen SPEAKER als Frame Element), sind sie doch unterschiedlich und werden daher auch von unterschiedlichen „Lexical Units“ (Wortbedeutungen) evoziert (vgl. Boas 2005b, Boas 2010a).

10 Siehe http://framenet.icsi.berkeley.edu/ (Stand: 25.10.2010). 
Der Statement Frame wird nicht nur vom Verb talk evoziert, sondern auch von anderen bedeutungsverwandten Verben wie z.B. claim oder contend, sowie von Substantiven wie z.B. declaration und statement. Die frame-semantische Beschreibung von Wortbedeutungen hat eine Reihe von Vorteilen. Erstens erlaubt sie eine systematische Analyse der zum Verstehen eines Wortes notwendigen Bedeutungsstrukturen und der dazugehörigen Konzepte, die auch für eine adäquate Verwendung von Wörtern notwendig sind. Zweitens ermöglicht sie es, bedeutungsverwandte Wörter mit demselben Beschreibungsinventar zu analysieren, auch über Wortartengrenzen hinweg. Drittens schafft eine solch feingegliederte Strukturierung des Lexikons auch die Grundlage, die Beziehungen zwischen der Bedeutung eines Wortes und ihrer unterschiedlichen syntaktischen Realisierung systematisch zu erfassen. So enthält z.B. der FrameNet-Eintrag des Verbs talk nicht nur Informationen über die den statement Frame konstituierenden Frame Elements, sondern auch eine ausführliche auf Korpusdaten basierende Auflistung der syntaktischen Realisierungen der Frame Elements (siehe Fillmore 2007, 2009). ${ }^{11}$ Der Hauptgedanke dieser Art der frame-semantischen Strukturierung des Lexikons ist es, Wörter so genau wie möglich nach unterschiedlichen Bedeutungsstrukturen und Konzepten zu beschreiben und darzustellen, wie die Bedeutungen einzelner Wörter syntaktisch unterschiedlich realisiert werden können.

Wenn man nun die von Fillmore konzipierte frame-semantische Strukturierung des Lexikons mit der von Goldberg $(1995,2006)$ vergleicht, so fallen ein paar wichtige Unterschiede auf. Erstens folgt Goldberg nicht genau der detaillierten Beschreibungsmethode von Fillmore bzgl. der Struktur und Detailgenauigkeit von Frames. Zwar behauptet sie, dass lexikalische Einträge auf Weltwissen zugreifen (,lexical entries should make reference to world and cultural knowledge", Goldberg 1995, S. 28), aber die Umsetzung dieses Gedankens scheint nicht sehr gelungen, wie der Lexikoneintrag des Verbs talk, in (2) zeigt. Goldberg räumt zwar ein, dass es schwierig ist frame-semantisches Wissen systematisch zu beschreiben, aber ihre eigenen Lexikoneinträge gehen auch nicht über die Strukturen und Inhalte wie in (2) hinaus. Eine Interpretation der Informationen in (2) mag zwar Auskunft darüber geben, dass es eine Person gibt, die spricht (die Partizipantenrolle TALKER), aber sonst wird nichts weiter über die Situation, andere Beteiligte, oder die begleitenden Umstände gesagt. Im Vergleich zu Fillmores

$11 \quad$ Ein weiterer Vorteil frame-semantischer Analysen ist die Tatsache, dass sich die für das Englische entwickelten Frames auch weitgehend auf die Analyse von anderen Sprachen übertragen lassen. Diese Methode erlaubt konstrastive Studien auf semantischer Ebene (d.h. der Frame-Ebene), wobei die syntaktischen Realisierungen der Frame Elements als die wirklich idiosynkratischen Eigenschaften einzelner Sprachen betrachtet werden können. Siehe z.B. die in Boas (Hg:) (2009) enthaltenen Beiträge über FrameNets für Deutsch, Japanisch, Spanisch, Hebräisch und Französisch. 
etablierter Framekonzeption lässt sich hier also eine stark reduzierte Konzeption der Frame-Semantik feststellen, die sonst keine weiteren Auskünfte gibt und offen für sehr unterschiedliche Interpretationen sein mag (siehe auch Boas 2003a, 2007, 2008b; Iwata 2008).

Zweitens erscheint Goldbergs Einbeziehung der Frame-Semantik in Bezug auf Polysemiestrukturen von Wörtern problematisch (siehe auch Kay 1996; Nemoto 1998; Boas 2002b, 2008c). Im Gegensatz zu Fillmore, dessen FrameNet klar veranschaulicht, dass unterschiedliche Wortbedeutungen unterschiedliche semantische Frames aktivieren, scheint Goldberg von einer eher minimalistischen Sichtweise von Verbbedeutung auszugehen. So ist außer den Informationen über die Partizipantenrolle TALKER in (2) nichts weiter über mögliche andere Bedeutungen von talk, zu erfahren, die eventuell auch für eine mögliche Fusionierung mit Konstruktionen in Frage kommen könnten. Andererseits zeigt die frame-semantische Repräsentation von talk, in FrameNet, dass es außer dem statement Frame auch noch die Frames Chatting und Talking_into aktivieren kann, welche sich trotz ihrer semantischen Verwandschaft deutlich voneinander unterscheiden (vgl. die Definitionen in FrameNet sowie die des zur Beschreibung von Frames verwendeten Formalismus, um auch Konstruktionen beschreiben zu können (siehe hierzu Fillmore 2008)). ${ }^{12}$

Goldbergs Ansatz unterscheidet sich von Fillmores Theorie in einem weiteren wichtigen Punkt. Während in der Frame-Semantik darauf geachtet wird, dass das so genannte Linking von semantischen Argumenten (den Frame Elements) zur Syntax hin zu einem gewissen Grad als eine vom Verb alleine bestimmte Eigenschaft angesehen wird (vgl. z.B. Boas 2003b, i.Dr.), schlägt Goldberg (1995, S. 28) das Gegenteil vor:

Since the mapping between semantics and syntax is done via constructions, not via lexical entries, that there should be a class of „syntactically relevant aspects of verb meaning" follows from the existence of constructions, which are independently motivated.

\subsection{Minikonstruktionen und konventionalisierte Resultative}

Um die Probleme mit Goldbergs Implementierung der Frame-Semantik zu lösen, habe ich die so genannte Ereignis-basierte Frame-Semantik (Boas 2003a, S. 159-213) entwickelt, die im Prinzip mit Goldbergs Ansatz kompatibel ist, aber mit viel größerer Genauigkeit die unterschiedlichen semantischen, pragmatischen und syntaktischen Anforderungen von einzelnen so genannten Minikonstruktionen (= Verbbedeutungen) beschreibt, die zum großen Teil die für die Lizenzierung von Resultativkonstruktionen wichtigen Informationen enthalten. Der zentrale Gedanke ist, dass jede einzelne

Goldberg würde wohl eine TALK-INTO-Konstruktion annehmen, in die das monoseme Verb talk eingesetzt würde. 
Bedeutung eines Verbs eine so genannte Minikonstruktion bildet, d.h. eine Paarung von Form mit Bedeutung/Funktion. Im Gegensatz zu Goldbergs Ansatz, der eine Lizenzierung von Resultativen durch die Fusionierung von abstrakt-schematischen Resultativkonstruktionen mit minimalen Lexikoneinträgen von Verben erklärt, sieht der in Boas (2003a) vertretene Alternativansatz vor, dass Resultative in der Sprachproduktion direkt von konventionalisierten Minikonstruktionen lizenziert werden, die ihre eigenen spezifisch-idiosynkratischen Restriktionen mit sich bringen. Der semantische Teil einer Minikonstruktion (der so genannte Ereignis-Frame) erfasst in größtmöglicher Genauigkeit den semantischen Frame, der von einer Verbbedeutung evoziert wird. Dieser Frame wird aber nicht nur mit den traditionell frame-semantischen Methoden wie oben dargestellt beschrieben, sondern wird um weitere relevante Informationen spezifiziert. In Anlehnung an Talmys (2000) so genannte „Force Dynamics“ werden frame-semantische Beschreibungen einzelner Verbbedeutungen um detailliertere Informationen über die einzelnen Frame Elements, ihre Beziehungen zueinander und den zeitlichen Ablauf des von der Verbbedeutung beschriebenen Geschehens erweitert.

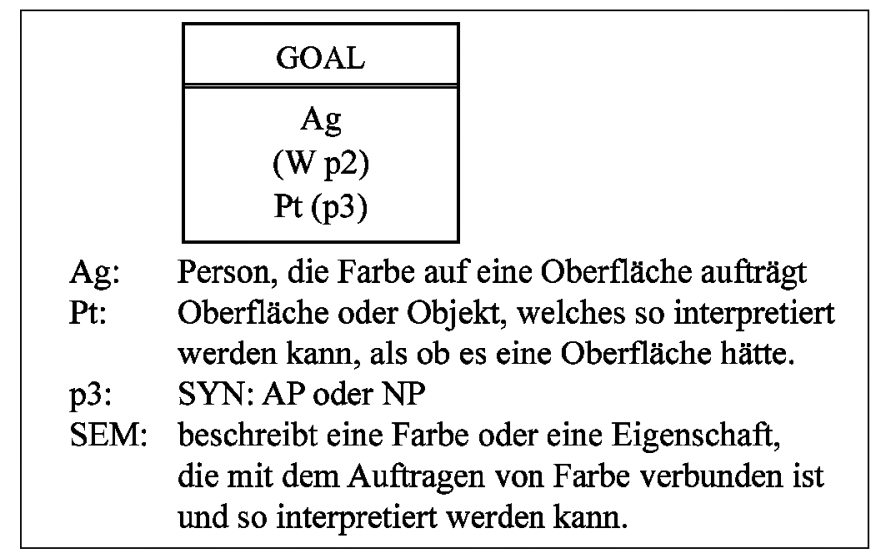

Abb. 3: Ereignis-basierte frame-semantische Repräsentation der prototypischen Bedeutung von paint, inkl. Weltwissen (W) (vgl. Boas 2003a, S. 224)

Als Beispiel sei hier die prototypische Bedeutung des Verbs paint (,malen ${ }^{c}$ bzw. ,bemalen') genannt, die ein Ereignis evoziert, in dem ein AGENT einem PATIENT Farbe aufträgt. Das Ereignis fängt zu einem bestimmten Zeitpunkt an (SOURCE), zieht sich über die Zeitspanne des Malens hin (PATH) und kommt letztendlich zum Abschluss, wenn das Ziel (GOAL) des Malens erreicht ist. Ein Teil der Bedeutung des Verbs ist also - neben den einzelnen Frame Elements - auch das Wissen, dass sich Ereignisse über bestimmte Zeitspannen ereignen und zu einem Endzustand führen kön- 
nen. Dieser Endzustand des von paint bezeichneten Ereignisses ist in Abbildung 3 dargestellt, wo nur der letzte Zeitpunkt des Ereignisses (GOAL) und der Endzustand der Frame Elements dargestellt ist (SOURCE und PATH, die den Zeitraum vor GOAL darstellen, sind ausgelassen, weil hier irrelevant).

Der Ereignis-Frame in Abbildung 3 repräsentiert die prototypische Bedeutung von paint bzw. den Bedeutungsteil der Minikonstruktion (der Formteil wird unten besprochen). Unterhalb des Kästchens in Abbildung 3 befinden sich genauere Spezifikationen der Frame Elements. Sie spezifizieren die semantischen und pragmatischen Selektionsrestriktionen des AGENT $(\mathrm{Ag})$ und des PATIENT (Pt), sowie mögliche Endresultatszustände (p2, p3), die aus der von paint beschriebenen prototypischen Aktivität hervorgehen können. Die Spezifizierung von p3, dem Endresultatszustand des PATIENT, zeigt darüber hinaus auch, dass neben semantischen und pragmatischen Restriktionen auch syntaktische Restriktionen im Ereignis-Frame festgelegt werden können. So ist die syntaktische Kategorie der Resultativphrase auf Nominalphrasen (z.B. a light shade of blue) und Adjektivphrasen (z.B. red) festgelegt. Die eingeklammerten Elemente p3, W, und p2 sind optional, d.h., sie müssen nicht syntaktisch realisiert werden (aber sie können implizit mitverstanden werden). Die durch Minikonstruktionen erfassten Spezifikationen sind als Defaultspezifikationen zu verstehen, d.h. sie können ggf. von anderen grammatischen Konstruktionen im gegebenen Kontext modifiziert werden. W steht für das übrige Weltwissen, welches für die Benutzung des Verbs paint relevant sein kann, z.B. dass man häufig Pinsel zum Malen benutzt, oder dass man müde werden kann, wenn man sehr lange malt. Das von W repräsentierte Weltwissen ist häufig irrelevant, manchmal kann es aber relevant werden, wenn nichtprototypische Endresultatszustände von Malaktivitäten beschrieben durch p2 ausgedrückt werden sollen wie z.B. Joe painted the brush to pieces (Joe malte die Bürste kaputt) oder Miriam painted herself tired (Miriam malte sich müde) (vgl. Boas 2003a, S. 181-192, 224, 256-259). ${ }^{13}$

Die so durch den Ereignis-Frame bereitgestellten frame-semantischen Informationen und Kollokationsrestriktionen sind nicht nur viel detaillierter in ihren Strukturen und Inhalten als die von Goldberg vorgeschlagenen Lexikoneinträge, sondern werden auch durch generelle Linking Rules à la Dowty (1991) direkt zur syntaktischen Realisierung gebracht (vgl. Boas 2003a, S. 244, 290). So lizenziert der Ereignis-Frame in Abbildung 3 Sätze

13 Vgl. z.B. Charniak (1977), der ausführlich die beim Malen eines Bildes mitverstandenen Informationen bespricht. Es ist auch möglich festzulegen, welche Ereignis-Frames besondere Arten von Resultativsätze lizenzieren können, wie z.B. so genannte „, fake object resultatives“" Diese Informationen können in den Ereignis-Frames detailliert festgelegt werden, um so die Übergenerierung wie in (4) und (6)-(9) oben zu verhindern. Die speziellen Lizenzierungsbedingungen für ,fake object resultatives ${ }^{c c}$ sind in Boas (2003a, S. 181-224, 240-260) dargestellt. 
wie Collin painted the barn red (,Collin malte die Scheune rot an): Der AGENT wird als Subjekt realisiert, der PATIENT als Objekt, und der optionale Endresultatszustand wird als adjektivische Resultativphrase realisiert. In diesem Zusammenhang muss klargestellt werden, dass der in Abbildung 3 dargestellte Ereignis-Frame nur einer von mehreren Ereignis-Frames von paint ist, nämlich der prototypische.

Der Vorteil dieser detaillierten Ereignis-Frames liegt darin, dass sie die oben diskutierten Probleme mit Goldbergs Ansatz lösen können. So kann nicht nur genau spezifiziert werden, welche Arten von Beziehungen es zwischen den einzelnen Frame Elements eines Ereignis-Frames gibt, sondern es ist auch möglich, dass Kollokations- und Frequenzinformationen genau spezifiziert werden können, sowohl für die postverbale Nominalphrase als auch für die Resultativphrase. Da diese Analyse auf Korpusdaten basiert, ist es möglich, die Kollokationsrestriktionen unterschiedlich genau zu fassen, d.h. von sehr generell wie „Oberfläche ${ }^{c}$ in Abbildung 3, bis zu genaueren Restriktionen wie ,„NP oder $\mathrm{AP}^{\prime \prime}$, bis hin zu sehr detaillierten Restriktionen, die genau Auskunft darüber geben können, welche Resultativphrasen lizenziert werden können, und welche nicht (vgl. Tabelle 2 und 3 oben, sowie Boas 2003a, S. 321-343). Die Möglichkeit, genaue Spezifikationen unterschiedlicher Art und Granularität auf der Ebene der Ereignis-Frames (dem Bedeutungsteil einer Minikonstruktion) vorzunehmen, ermöglicht es auch, die unterschiedliche und z.T. idiosynkratische Verteilung von Resultativen bei bedeutungsnahen Verben zu erfassen.

\subsection{Nichtkonventionalisierte Resultative: Lizenzierung durch Analogie}

Soweit bezieht sich mein Vorschlag nur auf konventionalisierte EreignisFrames, d.h. solche, die auf Grund von attestierten Korpusdaten klar identifiziert und als solche kodifiziert werden können. Wie aber steht es mit Goldbergs Paradebeispielen wie Joe conghed the napkin off the table (,Joe hustete die Serviette vom Tisch), die u.a. als Hauptargumente für die Existenz von unabhängig existierenden (abstrakten) Konstruktionen angeführt werden? In ihrer Kritik behaupten Goldberg/Jackendoff (2004, S. 562), dass meine Analyse in Boas (2003a) nicht den Daten gerecht wird, weil sie angeblich keine Möglichkeit vorsieht, neuartige nichtkonventionalisierte Resultativkonstruktionen zu lizenzieren. Sie argumentieren, dass nur eine abstrakte bedeutungstragende Resultativkonstruktion in der Lage sei, nichtkonventionalisierte Resultative durch Fusionierung mit Lexikoneinträgen zu lizenzieren. Doch in ihrer Kritik haben Goldberg/Jackendoff augenscheinlich einen wichtigen Teil übersehen, der sich eben auch mit solchen Fällen beschäftigt.

Mein Alternativvorschlag, dargestellt in Boas (ebd., S. 260-277), nimmt nämlich auch an, dass nichtkonventionalisierte Resultative auf der Basis von Analogie mit bereits existierenden konventionalisierten Resultativen lizen- 
ziert werden können. Die Idee ist, dass es z.B. Verben wie blow (blasen') gibt, die bereits die in ihren Ereignis-Frames spezifizierten semantischen Informationen (,etwas durch blasen bewegen ") konventionell als [NP V NP PP] realisieren, wie in Joe blew the napkin off the table (Joe blies die Serviette vom Tisch).

Genauer gesagt: Wenn es eine neue Situation gibt, in der ein Verb wie cough in einer nichtkonventionalisierten Weise verwendet wird, um Bewegung eines Objekts durch Luftausstoß auszudrücken, dann können Sprecher von bereits existierenden konventionalisierten Minikonstruktionen Gebrauch machen, die diese Bedeutung (als Ereignis-Frame dargestellt) bereits konventionalisiert auf der syntaktischen Ebene als [NP V NP PP] realisieren, in diesem Falle blow. Gibt es eine solche prototypische Minikonstruktion, so kann ein Verb wie congh auch mit nichtkonventionalisierten syntaktischen Konstituenten realisiert werden, soweit sich die kontextuellen Gegebenheiten der jeweiligen Situationen zwischen blow und cough ausreichend überlappen (genaue Einzelheiten sind in Boas 2003a, S. 270-277 dargestellt). Der springende Punkt ist, dass im Falle einer analogischen Erweiterung der Bedeutung eines Verbes wie cough die ganze Bandbreite von semantischen, pragmatischen und syntaktischen Restriktionen eines so bereits konventionalisierten Verbs mit übernommen werden. ${ }^{14}$

Dieser Vorgang kann dann auch im Laufe der Zeit zu dem von Tomasello beschriebenen Prozess der Schematisierung führen:

Wenn Menschen wiederholt, ähnliche ${ }^{\iota}$ Dinge in ,ähnlichen` Situationen sagen, entwickelt sich daraus mit der Zeit ein sprachliches Verwendungsmuster, das in den Köpfen der Benutzer als neue Kategorie oder Konstruktion schematisiert wird mit unterschiedlichen Abstraktionsgraden. (Tomasello 2006, S. 21)

Der auf Analogie beruhende Prozess der Bedeutungserweiterung von Verben wie blow und cough und die daraus folgende Erweiterung der syntaktischen Möglichkeiten lässt sich auch auf sprachhistorischer Ebene belegen. So paart z.B. das Verb blow laut Oxford English Dictionary seit 1382 die Bedeutung „etwas durch Luftausstoß bewegen zu können“ mit dem syntaktischen Rahmen [NP V NP PP]. Andere Verben wie cough und sneere, die in ihrer Grundbedeutung ebenfalls wie blow starken Luftausstoß beschreiben können, sind aber (noch nicht) mit der erweiterten Bedeutung konventionalisiert. ${ }^{15}$

Ein entscheidender Vorteil meiner Methode ist, dass man so auch bei nichtkonventionalisierten Resultativen die relevanten Restriktionen auf der Ebene der Minikonstruktionen festhalten kann. Da durch analogische Asso-

14 Welke (2009a, S. 517-524) schlägt eine parallele Vorgehensweise im Rahmen der Valenzgrammatik vor, um so z.B. Valenzerweiterungen von Verben zu analysieren.

$15 \mathrm{Zu}$ ähnlichen Vorschlägen in der diachronen Sprachwissenschaft siehe u.a. Diewald (2006, 2008) und Traugott (2008). 
ziation die Restriktionen von z.B. blow weitgehend auf cough übertragen werden, ist es so möglich, auch diese nichtkonventionalisierten Resultative dementsprechend zu restringieren. Dies ist bei Goldberg/Jackendoff (2004) nicht möglich, da die von ihnen postulierten abstrakten Resultativkonstruktionen (ähnlich wie bei Goldberg 1995) nicht in der Lage sind, inakzeptable Lizenzierungen auszuschließen (siehe Boas 2005a, 2007). ${ }^{16}$

\subsection{Konstruktionsnetzwerke}

Auf den ersten Blick erscheinen die von Goldberg $(1995,2006)$ und von Boas (2003a, 2005a) vertretenen Analysen nicht miteinander kompatibel zu sein. Dem ist jedoch nicht so. Bei genauerem Hinsehen fällt auf, dass die beiden Vorschläge sehr wohl miteinander kompatibel sind. Der größte Unterschied zwischen den beiden Ansätzen liegt darin, welchen Einfluss man abstrakten Konstruktionen wie der Resultativkonstruktion zugesteht. So ergeben sich meines Erachtens aus der Perspektive des Hörers keine Probleme beim Dekodieren, d.h. dem Verstehen eines Resultativsatzes auf der Basis von Goldbergs minimalen Lexikoneinträgen in Verbindung mit der abstrakten Resultativkonstruktion. Ob konventionalisierte oder nicht konventionalisierte Resultative, alles, was ein Hörer bei der Interpretation eines unbekannten Resultativsatzes machen muss, ist, das Gehörte zu interpretieren, indem er es mit existierenden Verbeinträgen (die auch relativ minimal sein können) in Verbindung mit Resultativkonstruktion abgleicht. Die oben angesprochenen Kritikpunkte an Goldbergs Ansatz scheinen beim Verstehen von Resultativsätzen also nicht so gravierend zu sein.

Dieser Vorgang des Dekodierens unterscheidet sich jedoch prozedural und inhaltlich vom Vorgang des Kodierens, den ein Sprecher bei der Produktion eines Resultativsatzes durchführt. So sollte ein Sprecher nämlich von vornherein wissen, was für postverbale Konstituenten mit welchen Verben in Resultativsätzen erscheinen können. Auch das Wissen der bei der Produktion wichtigen Kollokationsrestriktionen ist entscheidend dafür, ob ein Sprecher einen akzeptablen Resultativsatz produzieren kann. Diese Situation ist ähnlich der eines Fremdsprachenlerners, der zwar sowohl die Einzelbedeutungen von Adjektiven wie kalt und Adverbien wie bitter als auch die Regel der Adjektivmodifikation kennen mag, um so Adjektivmodifikationen wie richtig gut, bitter kalt und schrecklich langweilig produzieren zu

16 Konventionalisierte Minikonstruktionen sind in meinem Ansatz als Basis für analogische Neubildungen zu verstehen. So kann eine Neubildung wie Es kamn ja nicht angeben, dass er Ibnen das Essen zom Teller bechelt (ein Hundetrainer kommentiert das Verhalten eines Hundes) dann entstehen, wenn das Verb becheln per Analogie zu blasen oder busten im Kontext so interpretiert werden kann, dass das postverbale Objekt vom Subjekt in der jeweiligen Situation durch einen starken Luftaussto B bewegt werden kann. Ich danke Wolfgang Imo für das Korpusbeispiel. $\mathrm{Zu}$ näheren Einzelheiten der Analogiebildung auf Grundlage von konventionalisierten Minikonstruktionen und kontextuellem Hintergrundwissen siehe Boas (2003a, S. 272-284). 
können, aber dennoch gleichzeitig wissen muss, dass man im Deutschen bitter kalt sagt, und nicht salzig kalt oder sauer kalt. Mit anderen Worten: Es gibt keine abstrakte Konstruktion in der Grammatik, die einem Fremdsprachensprecher systematisch helfen könnte, alleine die richtige Adjektivmodifikation zu wählen, um so die akzeptierte Modifikation von kalt durch bitter adäquat zu produzieren. Dies hat zur Folge, dass der Fremdsprachenlerner nicht nur die Regel der Adjektivmodifikation kennen muss, sondern auch die konventionalisierte Phrase bitter kalt als konkret-spezifizierten Extraeintrag in sein Lexikon mit aufnehmen muss.

Mit der Resultativkonstruktion verhält es sich ähnlich, d.h. um einen akzeptablen Resultativsatz produzieren zu können, muss ein Sprecher wissen, welche Sorten von postverbalen Konstituenten mit welchen Verben in Resultativsätzen vorkommen können. Dazu gehört, wie oben ausgeführt, nicht nur genaues Wissen über die Situationen, die von bestimmten Einzelbedeutungen von Verben beschrieben werden können (die so genannten Ereignis-Frames), sondern auch Informationen über semantische, pragmatische und syntaktische Restriktionen in Verbindung mit genauen Kollokationsrestriktionen und Frequenzinformationen. Ohne solch ein Wissen ist es meines Erachtens nicht möglich, die oben besprochenen Schwierigkeiten bzgl. des von Goldberg überbewerteten Einflusses der abstrakt-schematischen Resultativkonstruktion zu lösen.

Diese feinen Unterschiede zwischen Dekodierung und Kodierung mögen vielleicht auf den ersten Blick unwichtig erscheinen, werden aber noch immer in der Literatur übersehen oder falsch interpretiert. So stellt z.B. Welke (2009a, S. 516) fest: „Folgerichtig geht die Verallgemeinerung bei Goldberg (1995) induktiv vor sich, von unten (bottom up), vom Einzelnen zum Allgemeinen, und nicht deduktiv, von oben (top down), vom Allgemeinen zum Einzelnen." Meine Ausführungen haben, so hoffe ich, klar gezeigt, dass dem nicht so ist: Bei Goldberg (ebd.) und Goldberg/Jackendoff (2004) findet nämlich keine wirkliche „bottom up“-Verallgemeinerung statt, die aussagekräftige Vorhersagen über die Produktion von Resultativsätzen machen kann, weil sie ,nur c" abstrakte Konstruktionen in Verbindung mit unterspezifizierten Lexikoneinträgen postulieren.

Wie kann diesen Gegensätzen nun Rechnung getragen werden? Zunächst sollte bedacht werden, dass zahlreiche Analysen der letzten zwanzig Jahre überzeugende Argumente geliefert haben, die für eine Architektur von Lexikon, Syntax, und Semantik auf Netzwerkebene plädiert haben. So schlagen u.a. Bybee $(1985,2007)$, Langacker $(1987,2000)$, Barlow/Kemmer (Hg.) (2000) und Boas (2003a) einen so genannten ,usage-based"“-Ansatz vor, der auf der zentralen Rolle der Sprechererfahrung bei der Kategorisierung von linguistischen Informationen beruht. Dieser Ansatz vertritt die Auffassung, dass einzelne konkrete Tokens im täglichen Sprachgebrauch benutzt werden und als solche auch abgespeichert werden können, obwohl sie eine 
konkrete Realisierung abstrakterer Types (oder Schemata) darstellen. Bei hoch frequentem Gebrauch eines bestimmten Typus von Token kann nach Bybee (2003) eine Abstraktion erfolgen, die dann zu einer Generalisierung führt, was auf einer abstrakteren Ebene zur Emergenz eines abstrakten Schemas führen kann. Der entscheidende Punkt dabei ist die Tatsache, dass abstrakte Schemata zusammen mit ihren konkreten Tokens ein Netzwerk bilden können, in dem (auch häufig redundante) Informationen auf unterschiedlichen Abstraktionsebenen festgehalten werden können. Abbildung 4, in Anlehnung an Langackers (2000, S. 34) Diskussion der „Ditransitive Construction", stellt einen Ausschnitt eines solchen Netzwerks für Resultativkonstruktionen unterschiedlichen Abstraktionsgrads dar.

Abbildung 4 repräsentiert einen Ausschnitt des Netzwerks der Resultativkonstruktion mit unterschiedlichen Abstraktsionsebenen, wobei jede konkretere Ebene Informationen von den darüber liegenden Ebenen erbt. Der Formalismus ist an Goldberg (1995) angelehnt und soll darstellen, wie die Bedeutung einer Konstruktion mit einer bestimmten Form gepaart sein kann. Ebene I repräsentiert den höchsten Grad der Konstruktionsgeneralisierung bzgl. der Resultativkonstruktion, da sie keine eigenständige Bedeutung repräsentiert (dargestellt durch die $\varnothing$ auf der Konstruktionsbedeutungsebene). Die relativ bedeutungsleere Konstruktion auf Ebene I ist auf der abstrakt-schematischsten Stufe dieses Konstruktionsnetzwerksausschnitts und verbindet ein bestimmtes synktaktisches Muster $[[\mathrm{NP}][\mathrm{V}][\mathrm{NP}][\mathrm{XP}]]$ mit einer relativ leeren Semantik, d.h., sie spezifiziert nur, dass die erste Nominalphrase als AGENT (,, $\left.\mathrm{Ag}^{\mathrm{c}}\right)$ realisiert wird. Diese relativ unterspezifizierte Paarung von Form und Bedeutung ist eine abstrakt-schematische sekundäre Prädikationskonstruktion, die ihre Informationen nicht nur an Resultativkonstruktionen, sondern auch an andere Typen von sekundären Prädikationskonstruktionen (hier durch einen Pfeil nach rechts repräsentiert) wie Small Clause-Komplemente und -Adjunkte (vgl. Staudinger 1997) vererben kann.

Auf Ebene II befindet sich eine stärker spezifizierte Konstruktion, nämlich die abstrakt-schematische Resultativkonstruktion, die Informationen von Ebene I erbt, aber gleichzeitig auch spezifischere Angaben bzgl. der Paarung von Form und Bedeutung macht. Diese Konstruktion ist spezifischer als die auf Ebene I befindliche abstraktere Konstruktion, da ihre Semantik nicht nur die CAUSE-BECOME-Bedeutung ausdrückt, sondern auch gleichzeitig festlegt, dass die postverbale NP als PATIENT interpretiert wird, und die postverbale XP als RESULT-GOAL.

Die einzelnen Resultativkonstruktionen auf Ebene III sind wiederum konkretere Instantiierungen der abstrakteren Konstruktion auf Ebene II. Sie unterscheiden sich hauptsächlich dadurch, dass sie die unterschiedlichen syntaktischen Kategorien der Resultativphrase als AP, PP oder NP spezifizieren. Ebenen I-III bilden die abstrakt-schematischen Ebenen 
der Resultativkonstruktion, die für die Lizenzierung von Resultativsätzen (mit)verantwortlich sind. Die auf diesen Netzwerkebenen spezifizierten Resultativkonstruktionen spiegeln keine phrasenstrukturellen Hierarchien wieder, weil sie nur die Defaultsatzstellung im Hauptsatz darstellen. Andere Wortstellungsvarianten werden durch die in Abbildung 4 dargestellten Resultativkonstruktionen in Verbindung mit anderen Konstruktionen wie der Relativsatzkonstruktion oder der Passivkonstruktion lizenziert (siehe Ackerman/Webelhuth 1998).

I:

II:

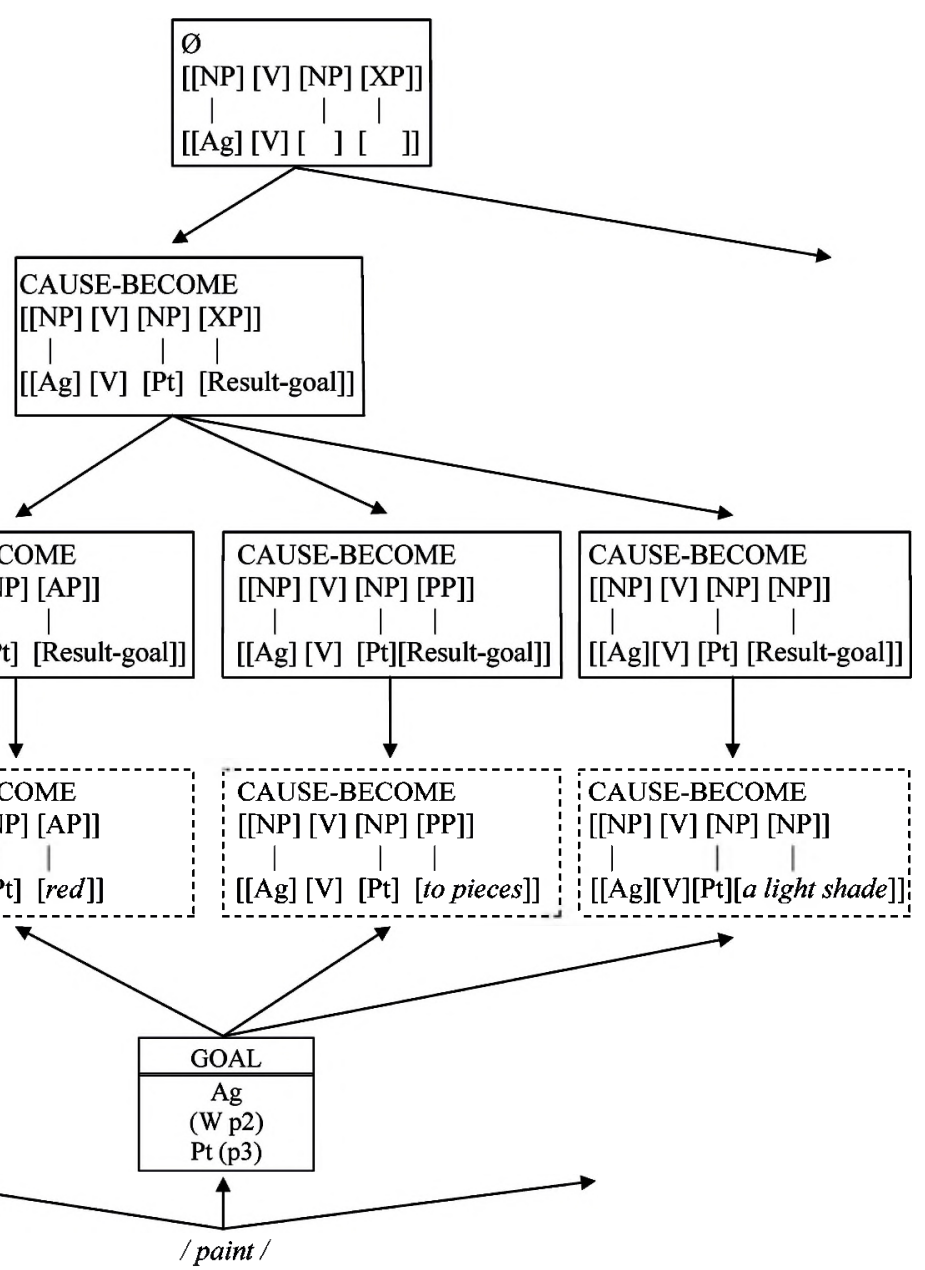

Abb. 4: Ausschnitt des Resultativkonstruktionsnetzwerks mit unterschiedlichen Abstraktionsgraden 
Auf der untersten Ebene IV finden wir eines der vielen Verben, die resultative Bedeutungen zum Ausdruck bringen können. Der Pfeil, der vom Verb paint auf den Ereignis-Frame zeigt, identifiziert die prototypische Bedeutung des Verbs, die hier durch einen die unterschiedlichen Partizipanten beschreibenden und andere Restriktionen festlegenden EreignisFrame festgehalten ist (vgl. Abbildung 2). Die anderen Pfeile zeigen auf hier nicht dargestellte nicht-prototypische Verbbedeutungen von paint, die wiederum durch andere Ereignis-Frames dargestellt werden können (vgl. Boas 2003a, S. 224-225). Ähnliche Verhältnisse lassen sich für andere Verben postulieren.

Die auf der so genannten Satzebene dargestellten Resultativkonstruktionen (mit gestrichelten Linien) zwischen Ebene III und Ebene IV bilden die Schnittmenge der auf Ebene III abstrakt-schematischen Konstruktionsschemata in Verbindung mit den durch den Ereignis-Frame lexikalisch-spezifizierten Informationen auf Ebene IV. Das bedeutet, dass durch die Satzebene lizenzierten Resultativsätze wie Joe paints the house red, Joe paints the brush to pieces und Joe paints the house a light shade of blue sowohl von abstrakt-schematischen Konstruktionen als auch von lexikalisch-spezifizierten EreignisFrames (Minikonstruktionen) lizenziert werden (siehe Boas 2003a, S. 240260). ${ }^{17}$ Mit anderen Worten: Resultativsätze werden gleichzeitig von der abstrakten Resultativkonstruktion und einem lexikalisch-spezifischen Ereignis-Frame lizenziert, der die idiosynkratischen Unterschiede zwischen einzelnen Verbbedeutungen festhält. ${ }^{18}$ Die Notwendigkeit der Arbeitsteilung von verbbasierten und konstruktionsbasierten Informationen wird auch von Welke im Rahmen seines Vergleichs von konstruktionsbasierten und valenzbasierten Ansätzen hervorgehoben:

Auch wenn man den Zusammenhang von Verb und Konstruktion von der Konstruktion aus angeht, muss man beide miteinander abgleichen. Beim Verb müssen Informationen über mögliche Konstruktionen mitgegeben sein und bei der Konstruktion über mögliche Verben. (Welke 2009b, S. 88)

17 Traugott (2008, S. 8) schlägt im Rahmen einer konstruktionsbasierten Grammatikalisierungstheorie ähnliche Prinzipien zur Erfassung von Konstruktionen unterschiedlichen Abstraktheitsgrads vor, die miteinander verwandt sind. So postuliert sie z.B. auch unterschiedliche Ebenen eines hierarchischen Systems, in dem unterschiedliche Konstruktionsebenen angenommen werden müssen: „(a) Makrokonstruktionen: abstrakte Schemata, die höchststufige Ebene, die für die gegebene Diskussion relevant ist, z.B. die Transitive Konstruktion, die Ditransitive Konstruktion, (...); (b) Mesokonstruktionen: Mengen sich ähnlich verhaltender Konstruktionen (...), (c) Mikro-Konstruktionen: Individuelle Konstruktionstypen [...]; und (d) Konstrukte: empirisch attestierte Token Gusage-based events' in der Terminologie von Langacker 1987), der Ort von Innovationen.“"

18 Konstruktionsnetzwerke werden auch erfolgreich für die Analyse von Spracherwerbsdaten (Tomassello 1998, 2003; Behrens 2009) und Grammatikalisierungsprozesse (Traugott 2008; Diewald 2009) eingesetzt. 
Das hier vorgeschlagene Netzwerkmodell, das Informationen von abstraktschematischen Konstruktionen mit denen aus Ereignis-Frames (Minikonstruktionen) kombiniert, folgt meines Erachtens dem von Welke gemachten Vorschlag.

Der hier skizzierte Alternativansatz, der die Analysen von Goldberg $(1995,2006)$ und Boas (2003a, 2005a) miteinander verbindet, mag durch die explizite Spezifizierung von Konstruktionsinformationen auf unterschiedlichen Abstraktionsebenen womöglich als zu unökonomisch kritisiert werden. So behauptet z.B. Müller (2006), dass zur Erfassung von Resultativkonstruktionen in der Konstruktionsgrammatik unnötig viele Annahmen gemacht werden müssen. Müller stützt seine Behauptung auf die Beobachtung, dass die Postulierung von komplexen Konstruktionen zu einer enormen Anzahl von weiteren konkreten komplexen Konstruktionen führen würde, um so allen Vorkommensmöglichkeiten von Resultativen in verschiedenen syntaktischen Bildungsweisen wie z.B. ,Scrambling", Passiv- und Mediumsätze, und Sätze mit Adverbialen Rechnung zu tragen (vgl. auch Jacobs 2008). Um das ganze Spektrum von Resultativen zu erfassen, müsse man mindestens 32 verschiedene komplexe Resultativkonstruktionen annehmen. Dies sei aber nicht plausibel, da man nicht davon ausgehen kann, dass Sprecher des Deutschen all diese 32 Konstruktionen einzeln im Langzeitgedächtnis gespeichert haben. Als Alternative schlägt Müller (2006) vor, dass eine einzige lexikalische Regel die von ihm besprochenen Daten erfassen könne.

Meine obigen Ausführungen stehen diesen Beobachtungen nicht unbedingt entgegen. So ist das Resultativkonstruktionsnetzwerk in Abbildung 4 so strukturiert, dass es auf abstrakt-schematischeren Ebenen wie Ebene I und II unterspezifizierte Repräsentationen gibt, die sich von anderen Ebenen unterscheiden. Auf diese Art werden bis zur Ebene III hinunter abstrakt-schematische Informationen bzgl. Resultativbildungen erfasst, die auch gleichzeitig mit Konstruktionen aus anderen Konstruktionsnetzwerken wie dem Passiv (vgl. Ackerman/Webelhuth 1998) fusionieren können, um den von Müller (2006) besprochenen syntaktischen Bildungsweisen Rechnung zu tragen. Die kognitiven Repräsentationen der unterschiedlichen Konstruktionen mögen zwar redundant sein, aber sie erfassen die umfangreichen zur Lizenzierung nötigen Spezifikationen. Die von mir als Minikonstruktionen bzw. Ereignis-Frames bezeichneten Einheiten auf der Satzebene halten demnach nur die minimalen Idiosynkrasien einzelner Verbbedeutungen fest, die unbedingt - da sie konventionalisiert sind - als eigenständige konkrete semantische, pragmatische und syntaktische Spezifizierungen von abstrakt-schematischeren Konstruktionen gelistet werden müssen (vgl. Abschnitt 2.3). Die obigen Vorschläge müssen natürlich noch verfeinert werden, besonders bzgl. der Arbeitsteilung zwischen abstraktschematischen und konkret-spezifischen Konstruktionen. 
3.4 Ereignis-Frames und abstrakte Konstruktionen im Englischen und Deutschen

Die oben dargestellten Vorschläge lassen sich auch auf das Deutsche weitgehend übertragen. ${ }^{19}$ So gibt es auch im Deutschen viele Verben, die sowohl der Struktur als auch der Bedeutung der oben besprochenen englischen Resultativbeispiele entsprechen:
a. Ursula wischte den Tisch (sauber).
b. Ulrich strich das Haus (rot).
c. Fritz lief sich müde.
a'. Ursula wiped the table (clean).
b'. Ulrich painted the house (red).
c'. Fritz ran himself tired.

(vgl. Boas 2003a, S. 286)

Doch der Schein trügt: Nicht wirklich alle deutschen Übersetzungen englischer Verben können auch im Deutschen in Resultativsätzen vorkommen. Die Beispiele in (11) und (12) zeigen, dass die deutschen Verben rennen und bungern nicht wie ihre englischen Entsprechungen run und starve in Resultativkonstruktionen vorkommen können. Das Gegenteil ist auch in (13) der Fall, wo das englische pray nicht wie beten in einer Resultativkonstruktion vorkommen kann.

(11) a. Martin ran Ed off the road.

b. *Martin rannte Ed von der Straße ab.

c. Martin drängte Ed (beim Rennen) von der Straße ab.

(12) a. The doctors starved the patients to death.

b. *Die Ärzte hungerten die Patienten zu Tode.

c. Die Ärzte ließen die Patienten verhungern.

(13) a. Der Mann betete seine Tochter gesund.

b. ?The man prayed his daughter healthy.

c. The man prayed. As a result of his praying, his daughter became healthy.

(vgl. Boas 2003a, S. 287)

Aus Daten wie in (11)-(13) lassen sich folgende Schlussfolgerungen ziehen: (1) Trotz ihrer parallelen Grundbedeutungen können nicht alle englischen Verben und ihre deutschen Entsprechungen in ihrer Defaultinterpretation in Resultativkonstruktionen vorkommen. (2) In einigen Fällen verlangen das Deutsche bzw. das Englische ein völlig anderes Verb in Verbindung mit einer anderen Konstruktion. Dies ist z.T. auf typologische Unterschiede zurückzuführen (z.B. die Tatsache, dass im Deutschen ein genauer Unterschied zwischen den Arten des Wissens, Verlassens oder Stellens gemacht

$19 \quad \mathrm{Zu}$ den Einzelheiten der hierbei relevanten Aspekte der deutschen Verbalmorphologie siehe u.a. Müller $(2002,2005,2006)$. 
werden muss, aber nicht im Englischen - vgl. Hawkins 1986; Leisi/Mair 1999; Boas 2003a), muss aber dennoch irgendwo in der Grammatik bzw. im Lexikon festgehalten werden. (3) Die Zusammensetzung von Verben und postverbalen Konstituenten in Resultativkonstruktionen muss im Deutschen genauso wie im Englischen durch so genannte Ereignis-Frames lizenziert werden, die als lexikalisch-spezifizierte Minikonstruktionen die einzelnen Unterbedeutungen von Verben repräsentieren und so als konkrete Spezifizierung einer abstrakten Resultativkonstruktion angesehen werden (vgl. Abschnitt 3.3). Ein weiterer Beleg für diese Sichtweise ergibt sich aus folgender Tabelle:

\begin{tabular}{|c|c|c|c|c|}
\hline & Bedeutung & $\begin{array}{l}\text { Englische } \\
\text { Resultativkonstruktion }\end{array}$ & $\begin{array}{l}\text { Deutsches } \\
\text { Äquivalent }\end{array}$ & $\begin{array}{l}\text { Deutsche } \\
\text { Resultativkonstruktion }\end{array}$ \\
\hline 1 & $\begin{array}{l}\text { To guide, con- } \\
\text { trol, or direct } \\
\text { (a vehicle) }\end{array}$ & $\begin{array}{l}\text { He drove the car into } \\
\text { the ditch/ to pieces. }\end{array}$ & fahren & $\begin{array}{l}\text { Er fuhr das Auto in } \\
\text { den Graben/kaputt. }\end{array}$ \\
\hline 2 & $\begin{array}{l}\text { To convey } \\
\text { transport in a } \\
\text { vehicle. }\end{array}$ & $\begin{array}{l}\text { She drove the kids to } \\
\text { school. }\end{array}$ & $\begin{array}{l}\text { befördern/ } \\
\text { fahren }\end{array}$ & $\begin{array}{l}\text { Sie beförderte/fuhr } \\
\text { die Kinder zur Schule. }\end{array}$ \\
\hline 3 & To penetrate & $\begin{array}{l}\text { Kim drove the stake } \\
\text { into the ground. }\end{array}$ & $\begin{array}{l}\text { (ein-)schlagen, } \\
\text { treiben }\end{array}$ & $\begin{array}{l}\text { Karla schlug den Stock } \\
\text { in den Boden (ein). }\end{array}$ \\
\hline 4 & $\begin{array}{l}\text { To repulse } \\
\text { forcefully; } \\
\text { put to flight }\end{array}$ & $\begin{array}{l}\text { We drove the attackers } \\
\text { away. }\end{array}$ & $\begin{array}{l}\text { wegjagen/ } \\
\text { vertreiben }\end{array}$ & $\begin{array}{l}\text { Wir jagten die An- } \\
\text { greifer weg./Wir ver- } \\
\text { trieben die Angreifer. }\end{array}$ \\
\hline 5 & $\begin{array}{l}\text { To throw, strike, } \\
\text { or cast }\end{array}$ & $\begin{array}{l}\text { He drove the ball into } \\
\text { the goal. }\end{array}$ & treten & $\begin{array}{l}\text { Er trat den Ball in das } \\
\text { Tor. }\end{array}$ \\
\hline 6 & $\begin{array}{l}\text { To change the } \\
\text { mental state of } \\
\text { somebody }\end{array}$ & She drove me crazy. & treiben & $\begin{array}{l}\text { Sie trieb mich in den } \\
\text { Wahnsinn. }\end{array}$ \\
\hline
\end{tabular}

Tab. 4: Englische Resultativkonstruktionen mit drive und ihre deutschen Entsprechungen (vgl. Boas 2003a, S. 295)

Das englische Verb drive verfügt über mindestens sechs unterschiedliche Bedeutungen, die alle in resultativer Verwendung vorkommen können. Im Gegensatz dazu enthalten die deutschen Übersetzungsäquivalente alle unterschiedliche Verben. Gemeinsam ist den englischen Beispielen und ihren deutschen Entsprechungen, dass die Ereignis-Frames genaue Kollokationsrestriktionen bzw. Spezifikationen bzgl. syntaktischer, semantischer und pragmatischer Restriktionen festlegen, die bei der Produktion eines Resultativsatzes nicht alleine durch eine abstrakte Resultativkonstruktion erfasst werden können (vgl. *Er fuhr das Auto grïn, * Sie fuhr die Kinder krank, * Sie trieb mich in die Gliicklichkeit, etc.). Daraus folgt u.a., dass sich die für das Englische vorgeschlagene Analyse von Resultativkonstruktionen im Rahmen von Ereignis-Frames auch weitgehend auf das Deutsche übertragen lässt. 


\title{
4. Schlussbetrachtungen
}

Das in diesem Beitrag vorgeschlagene Netzwerk aus Resultativkonstruktionen unterschiedlichen Abstraktionsgrads vereinigt die Vorteile von Goldbergs $(1995,2006)$ und Boas' $(2003 a, 2005 a)$ Analysen. So können sowohl die beim Dekodieren relevanten abstrakt-schematischen Konstruktionsinformationen als auch die beim Kodieren wichtigen lexikalisch-spezifizierten Konstruktionsinformationen über semantische, pragmatische, und syntaktische Restriktionen auf der Ebene von Ereignis-Frames in einem einheitlichen Netzwerk erfasst und analysiert werden. Dies erfüllt auch die von Goldberg (2006, S. 18) postulierte Prämisse: „It's constructions all the way down."

Diese einheitliche Analyse bietet auch zwei weitere entscheidende Vorteile. Erstens vereinfacht die Beschreibung der Distribution von Konstruktionen auf primär semantisch-pragmatischer Ebene die kontrastive Analyse von gleichartigen Konstruktionen in unterschiedlichen Sprachen. Da der Formteil einer Konstruktion letztendlich nur sekundären Rang in der Konstruktionsbeschreibung hat, ist es so einfacher, sprachvergleichend Konstruktionen zu beschreiben, wie in Abschnitt 3.4 gezeigt wurde (vgl. dazu Boas 2003a, 2010b). Zweitens erfüllt die hier vorgeschlagene Methode auch die in neueren Veröffentlichungen zu Parallelen der Valenzgrammatik und Konstruktionsgrammatik gemachten Vorschläge, unterschiedliche Abstraktionsgrade von Konstruktionen zu berücksichtigen (vgl. Jacobs 2008; Welke 2009a, b). Welke $z . B$. fordert:

\begin{abstract}
Wir plädieren, was die heutige Valenz- und Konstruktionsgrammatik betrifft, nicht für einseitige Übernahmen oder Abgrenzungen, sondern für eine Integration. Valenzänderungen (einschliesslich Valenzerweiterungen und Valenzreduktion) und Valenzvererbung können als konstruktionell bedingte Änderungen erklärt werden. Grundlage ist einerseits ein am Einzelwort, andererseits ein an der Einzelkonstruktion orientiertes Vorgehen, das nicht vom Allgemeinen zum Einzelnen gelangt, sondern via prototypischer und analogischer Verallgemeinerung vom Einzelnen zum Allgemeinen. Wesentlich ist in diesem Zusammenhang die Frage nach dem jeweils erreichten Verallgemeinerungsgrad, der „Reichweite“, von Konstruktionen. (Welke 2009b, S. 541)
\end{abstract}

Meine in diesem Beitrag skizzierten Vorschläge setzen meines Erachtens die von Welke (2009b) gestellten Integrationsforderungen bzgl. Wörtern und Konstruktionen in die Tat um. Zukünftige Analysen sollten sich noch eingehender mit der genauen Arbeitsteilung von Verben und Konstruktionen beschäftigen sowie systematische Untersuchungen zu Interaktionen zwischen unterschiedlichen Konstruktionen anstellen. Darüber hinaus ist auch die Entwicklung eines noch einheitlicheren Formalismus zur Beschreibung von Konstruktionen unterschiedlichen Abstraktionsgrads wie in der „Sign-based Construction Grammar" (Michaelis 2010; Sag 2010; Boas/Sag (Hg.) i.Dr.; Fillmore/Lee-Goldman/Rhodes i.Dr.) wünschenswert. 


\section{Literatur}

Ackerman, Farrell/Webelhuth, Gert (1998): A theory of predicates. Stanford.

Barðdal, Jóhanna (2008): Productivity: evidence from case and argument structure in Icelandic. (= Constructional Approaches to Language 8). Amsterdam/Philadelphia.

Barlow, Michael/Kemmer, Susanne (Hg.) (2000): Usage-based models of language. Stanford.

Behrens, Heike (2009): Konstruktionen im Spracherwerb. In: Zeitschrift für Germanistische Linguistik 37, S. 427-444.

Bergen, Benjamin/Chang, Nancy (2005): Embodied Construction Grammar in simulation-based language understanding. In: Östman/Fried (Hg.), S. 147-190.

Bergs, Alexander/Diewald, Gabriele (Hg.) (2008): Constructions and language change. Berlin/New York.

Boas, Hans C. (2002a): Lexical or syntactic selection of non-subcategorized nominal arguments? The case of German,Sätzchen'. In: Rauch, Irmengard/Carr, Gerald F. (Hg.): New Insights in Germanic Linguistics III. (= Berkeley Insights in Linguistics and Semiotics 52). New York, S. 9-26.

Boas, Hans C. (2002b): On the role of semantic constraints in resultative constructions. In: Rapp, Reinhard ( $\mathrm{Hg}$.): Sprachwissenschaft auf dem Weg in das Dritte Jahrtausend: Akten des 34. Linguistischen Kolloquiums in Germersheim 1999 (Linguistics on the way into the new millennium). Bd. 1. Frankfurt a.M., S. 35-44.

Boas, Hans C. (2003a): A constructional approach to resultatives. Stanford.

Boas, Hans C. (2003b): A lexical-constructional account of the locative alternation. In: Carmichael, Leslie/Huang, Scott C.-H./Samiian, Vida (Hg.): Proceedings of the 2001 Western Conference in Linguistics 13, S. $27-42$.

Boas, Hans C. (2004): You wanna consider a constructional approach to wanna-contraction? In: Achard, Michael/Kemmer, Suzanne (Hg): Language, Culture, and Mind. Stanford, S. 479-491.

Boas, Hans C. (2005a): Determining the productivity of resultative constructions: a reply to Goldberg \& Jackendoff. In: Language 81, 2, S. 448-464.

Boas, Hans C. (2005b): From theory to practice: Frame semantics and the design of FrameNet. In: Langer, Stefan/Schnorbusch, Daniel (Hg.): Semantik im Lexikon. (= Tübinger Beiträge zur Linguistik 479). Tübingen, S. 129-160.

Boas, Hans C. (2006): A frame-semantic approach to identifying syntactically relevant elements of meaning. In: Steiner, Petra/Boas, Hans C./Schierholz, Stefan (Hg.): Contrastive studies and valency. Studies in honor of Hans Ulrich Boas. Frankfurt a.M./New York, S. 119-149.

Boas, Hans C. (2007): Construction Grammar in the twenty-first century. In: English Language and Linguistics 11, 3, S. 569-585.

Boas, Hans C. (2008a): Determining the structure of lexical entries and grammatical constructions in Construction Grammar. In: Annual Review of Cognitive Linguistics 6, S. 113-144. 
Boas, Hans C. (2008b): Resolving form-meaning discrepancies in Construction Grammar. In Leino, Jaakko (Hg.): Constructional Reorganization. (= Constructional approaches to language 5). Amsterdam/Philadelphia, S. 11-36.

Boas, Hans C. (2008c): Towards a frame-constructional approach to verb classification. In: Acevedo, Eulalia Sosa/Rodríguez, Francisco José Cortés (Hg.): Grammar, constructions, and interfaces. Special Issue of Revista Canaria de Estudios Ingleses 57, S. $17-48$.

Boas, Hans C. (2009): Verb meanings at the crossroads between higher-level and lowerlevel constructions. In: Lingua 120, S. 22-34.

Boas, Hans C. (Hg.) (2009): Multilingual FrameNets in computational lexicography. Methods and applications. (= Trends in Linguistics: Studies and Monographs 200). Berlin/New York.

Boas, Hans C. (2010a): The syntax-lexicon continuum in Construction Grammar: a case study of English communication verbs. In: Belgian Journal of Linguistics 24, S. $57-86$.

Boas, Hans C. (2010b): Comparing constructions across languages. In: Boas, Hans C. (Hg.): Contrastive studies in Construction Grammar. Amsterdam/Philadelphia, S. $1-20$.

Boas, Hans C. (i. Dr.): A frame-semantic approach to syntactic alternations: the case of build verbs. In: Guerrero Medina, Pilar ( $\mathrm{Hg}$.): Morphosyntactic Alternations in English. London.

Boas, Hans C./Sag, Ivan A. (Hg.) (i.Dr.): Sign-based Construction Grammar. Stanford.

Bybee, Joan (1985): Morphology: a study of the relation between meaning and form. (= Typological Studies in Language 9). Amsterdam/Philadelphia.

Bybee, Joan (2003): Mechanisms of change in grammaticalization: the role of frequency. In: Joseph, Brian D./Janda, Richard D. (Hg.): The handbook of historical linguistics. Oxford, S. 602-623.

Bybee, Joan (2007): Frequency of use and the organization of language. Oxford.

Charniak, Eugene (1977). A framed PAINTING: the representation of a common sense knowledge fragment. In: Cognitive Science 1, S. 355-395.

Chomsky, Noam (1981): Lectures on government and binding. (= Studies in Generative Grammar 9). Dordrecht.

Chomsky, Noam (1995): The minimalist program. (= Current Studies in Linguistics Series 28). Cambridge, MA u.a.

Croft, William (2001): Radical Construction Grammar: syntactic theory in typological perspective. Oxford.

Deppermann, Arnulf (2007): Grammatik und Semantik aus gesprächsanalytischer Sicht. (= Linguistik - Impulse und Tendenzen 14). Berlin/New York.

Diessel, Holger (2004): The acquisition of complex sentences. (= Cambridge Studies in Linguistics 105). Cambridge.

Diewald, Gabriele (2006): Konstruktionen in der diachronen Sprachwissenschaft. In: Fischer/Stefanowitsch (Hg.), S. 79-103. 
Diewald, Gabriele (2008): Idiomatische Konstruktionen in Grammatikalisierungsprozessen. In: Stefanowitsch/Fischer (Hg.), S. 33-58.

Diewald, Gabriele (2009): Konstruktionen und Paradigmen. In: Zeitschrift für Germanistische Linguistik 37, S. 445-468.

Dowty, David (1991): Thematic proto-roles and argument selection. In: Language 67, S. 547-619.

Fillmore, Charles J. (1985): Frames and the semantics of understanding. In: Quadernie di Semantica 6, 2, S. 222-254.

Fillmore, Charles J. (1988): The mechanisms of Construction Grammar. In: Axmaker, Shelley/Jassier, Annie/Singmaster, Helen ( $\mathrm{Hg}$ ): General session and parasession on grammaticalization. Proceedings of the Fourteenth Annual Meeting of the Berkeley Linguistic Society. Berkeley, S. 35-55.

Fillmore, Charles J. (2007): Valency issues in FrameNet. In: Herbst, Thomas/Götz-Votteler, Katrin ( $\mathrm{Hg}$ ): Valency: theoretical, descriptive, and cognitive issues. (= Trends in Linguistics: Studies and Monographs 187). Berlin/New York, S. 129-160.

Fillmore, Charles J. (2008): Border conflicts: FrameNet meets Construction Grammar. Plenarvortrag, EURALEX XIII, Barcelona.

Fillmore, Charles J. (2009): A valency dictionary of English. In: International Journal of Lexicography 22, 1, S. 55-85.

Fillmore, Charles J./Atkins, Beryl T.S. (1992): Towards a frame-based organization of the lexicon: the semantics of RISK and its neighbors. In: Lehrer, Adrienne/Kittay, Eva (Hg): Frames, fields, and contrasts: new essays in lexical organization. Hillsdale, S. 75-102.

Fillmore, Charles J./Baker, Collin (2010): A frame approach to semantic analysis. In: Heine/Narrog (Hg), S. 313-340.

Fillmore, Charles J./Johnson, Christopher R./Petruck, Miriam R.L. (2003): Background to FrameNet. In: International Journal of Lexicography 16, 3, S. 235-250.

Fillmore, Charles J./Kay, Paul (1995): Construction Grammar. Ms. Berkeley.

Fillmore, Charles J./Kay, Paul/O'Connor, Mary (1988): Regularity and idiomaticity in grammatical constructions: The case of ,let alone 6 In: Language 64, S. 501-538.

Fillmore, Charles J./Lee-Goldman, Russell/Rhodes, Russell (i.Dr.): Sign-based Construction Grammar and the FrameNet constructicon. In: Boas/Sag ( $\mathrm{Hg}$.).

Fischer, Kerstin/Stefanowitsch, Anatol (Hg) (2006): Konstruktionsgrammatik. Von der Anwendung zur Theorie. (= Stauffenburg Linguistik 40). Tübingen.

Fried, Mirjam/Boas, Hans C. (Hg.) (2005): Grammatical constructions. Back to the roots. (= Constructional Approaches to Language 4). Amsterdam/Philadelphia.

Fried, Mirjam/Östman, Jan-Ola (Hg) (2004): Construction Grammar in a cross-language perspective. (= Constructional Approaches to Language 2). Amsterdam/ Philadelphia.

Goldberg, Adele (1995): Constructions: a construction grammar approach to argument structure. Chicago. 
Goldberg, Adele (2006): Constructions at work: the nature of generalization in language. Oxford.

Goldberg, Adele/Jackendoff, Ray (2004): The English resultative as a family of constructions. In: Language 80, 3, S. 532-568.

Gries, Stefan T./Wulff, Stefanie (2005): Do foreign language learners also have constructions? Evidence from priming, sorting, and corpora. In: Annual Review of Cognitive Linguistics 3, S. 182-200.

Günthner, Susanne (2008): Die "die Sache/das Ding ist"-Konstruktion im gesprochenen Deutsch - eine interaktionale Perspektive auf Konstruktionen im Gebrauch. In: Stefanowitsch/Fischer (Hg.), S. 157-178.

Hawkins, John (1986): A comparative typology of English and German. Unifying the contrasts. London.

Heine, Bernd/Narrog, Heiko (Hg.) (2010): The Oxford handbook of linguistic analysis. Oxford.

Hilpert, Martin (2008): Germanic future constructions: a usage-based approach to language change. (= Constructional Approaches to Language 7). Amsterdam/Philadelphia.

Imo, Wolfgang (2007): Construction Grammar und Gesprochene-Sprache-Forschung: Konstruktionen mit zehn matrixsatzfähigen Verben im gesprochenen Deutsch. (= Reihe Germanistische Linguistik 275). Tübingen.

Iwata, Seizi (2005): The role of verb meaning in locative alternations. In: Fried/Boas (Hg.), S. 101-118.

Iwata, Seizi (2008): Locative Alternation. A lexical-constructional approach. (= Constructional Approaches to Language 6). Amsterdam/Philadelphia.

Jackendoff, Ray (1997): The architecture of the language faculty. (= Linguistic Inquiry Monographs 28). Cambridge, MA.

Jackendoff, Ray (2010): Meaning and the lexicon: the parallel architecture, 1975-2010. Oxford.

Jacobs, Joachim (2008): Wozu Konstruktionen? In: Linguistische Berichte 213, S. 1-44.

Kay, Paul (1996): Argument structure: causative ABC constructions. Ms. University of California. Berkeley.

Kay, Paul (2005): Argument structure constructions and the argument-adjunct distinction. In: Fried/Boas (Hg.), S. 71-100.

Kay, Paul/Fillmore Charles J. (1999): Grammatical constructions and linguistic generalizations: the ,What's X doing Y?' constructions. In: Language 75, S. 1-33.

Lambrecht, Knud/Lemoine, Kevin (2005): Definite null objects in (spoken) French: A Construction-Grammar account. In: Fried/Boas (Hg:), S. 13-56.

Langacker, Ron (1987): Foundations of Cognitive Grammar I: Theoretical prerequisites. Stanford.

Langacker, Ron (2000): A dynamic usage-based model. In: Barlow/Kemmer (Hg.), S. $1-63$. 
Leisi, Ernst/Mair, Christian (1999): Das heutige Englisch: Wesenszüge und Probleme. 8., neubearb. Aufl. Heidelberg.

Michaclis, Laura (2010): Sign-based Construction Grammar. In: Heine/Narrog (Hg), S. 139-158.

Michaelis, Laura/Ruppenhofer, Joseph (2001): Beyond alternations: a constructional model of the German applicative pattern. Stanford.

Müller, Stefan (2002): Complex predicates: verbal complexes, resultative constructions, and particle verbs in German. Stanford.

Müller, Stefan (2005): Resultative constructions: syntax, world knowledge, and collocational restrictions. Review of Hans C. Boas: A Constructional Approach to Resultatives. In: Studies in Language 29, S. 651-681.

Müller, Stefan (2006): Resultativkonstruktionen, Partikelverben und syntaktische vs. lexikonbasierte Konstruktionen. In: Fischer/Stefanowitsch (Hg), S. 177-202.

Nemoto, Noriko (1998): On the polysemy of ditransitive save: the role of frame semantics in Construction Grammar. In: English Linguistics 15, S. 219-242.

Nemoto, Noriko (2005): Verbal polysemy and frame semantics in Construction Grammar. In: Fried/Boas (Hg.), S. 118-136.

Östman, Jan-Ola (2005): Construction discourse: a prolegomenon. In: Östman/Fried (Hg:), S. 121-144.

Östman, Jan-Ola/Fried, Mirjam (Hg.) (2005): Construction Grammars. Cognitive grounding and theoretical extensions. (= Constructional Approaches to Language 3). Amsterdam/Philadelphia.

Petruck, Miriam (1996): Frame semantics. In: Verschueren, Jef et al. (Hg): Handbook of pragmatics. Amsterdam/Philadelphia, S. 1-13.

Pollard, Carl/Sag, Ivan (1994): Head-driven phrase structure grammar. Stanford.

Ruppenhofer, Joseph et al. (2006): FrameNet II: extended theory and practice. Internet: http://framenet.icsi.berkeley.edu (Stand: 01.11.2010).

Sag, Ivan (2010): English filler-gap constructions. In: Language 86, 3, S. 486-545.

Staudinger, Bernd (1997): Sätzchen: Small clauses im Deutschen. (= Linguistische Arbeiten 363). Tübingen.

Stefanowitsch, Anatol/Fischer, Kerstin (Hg) (2008): Konstruktionsgrammatik II: Von der Konstruktion zur Grammatik. (= Stauffenburg Linguistik 47). Tübingen.

Talmy, Len (2000): Toward a cognitive semantics. Cambridge, MA.

Tomasello, Michael (1998): The return of constructions. In: Journal of Child Language 75 , S. 431-447.

Tomasello, Michael (2003): Constructing a language: a usage-based theory of language acquisition. Cambridge, MA.

Tomasello, Michael (2006): Konstruktionsgrammatik und früher Erstspracherwerb. In: Fischer/Stefanowitsch (Hg.), S. 19-38. 
Traugott, Elisabeth (2008): Grammatikalisierung, emergente Konstruktionen und der Begriff der „Neuheit". In: Stefanowitsch/Fischer (Hg.), S. 5-32.

van Trijp, Remi (2008): Argumentstruktur in der Fluid Construction Grammar. In: Stefanowitsch/Fischer (Hg.), S. 223-246.

Welke, Klaus (2009a): Valenztheorie und Konstruktionsgrammatik. In: Zeitschrift für Germanistische Linguistik 37, S. 81-124.

Welke, Klaus (2009b): Konstruktionsvererbung, Valenzvererbung und die Reichweite von Konstruktionen. In: Zeitschrift für Germanistische Linguistik 37, S. 514-543.

Ziem, Alexander (2008): Frames und sprachliches Wissen: kognitive Aspekte der semantischen Kompetenz. (= Sprache und Wissen 2). Berlin/New York. 